University of Wollongong

Research Online

Australian Institute for Innovative Materials -

Papers

Australian Institute for Innovative Materials

$1-1-2019$

\title{
Manipulation of the Electronic Transport Properties of Charge-Transfer Oxide Thin Films of NdNi 03 Using Static and Electric-Field-Controllable Dynamic Lattice Strain
}

\author{
Jian-Min Yan \\ Chinese Academy Of Sciences \\ Meng Xu \\ Chinese Academy Of Sciences \\ Ting-Wei Chen \\ Nanchang University \\ Ming Yang \\ University of Warwick \\ Fei Liu \\ Chinese Academy of Sciences
}

See next page for additional authors

Follow this and additional works at: https://ro.uow.edu.au/aiimpapers

Part of the Engineering Commons, and the Physical Sciences and Mathematics Commons

Research Online is the open access institutional repository for the University of Wollongong. For further information contact the UOW Library: research-pubs@uow.edu.au 


\title{
Manipulation of the Electronic Transport Properties of Charge-Transfer Oxide Thin Films of NdNi 03 Using Static and Electric-Field-Controllable Dynamic Lattice Strain
}

\author{
Abstract \\ Using perovskite-type charge-transfer oxide thin films of NdNiO3 (NNO) as a model system, we \\ demonstrate that the effects of lattice strain on the electronic transport properties can be more \\ comprehensively understood by growing NNO films on a number of (001)-, (011)-, and (111)-cut single- \\ crystal substrates with different lattice mismatches including the relaxor-based $0.31 \mathrm{~Pb}(\ln 1 / 2 \mathrm{Nb} 1 /$ \\ 2)03-0.35Pb(Mg1/3Nb2/3)03-0.34PbTiO3 (PIN-PMN-PT) and 0.71Pb(Mg1/3Nb2/3)03-0.29PbTiO3 \\ (PMN-PT) ferroelectric (FE) single crystals. In addition to the static lattice strains from conventional \\ substrates (e.g., SrTiO3, LaAlO3), we in situ impose in-plane compressive or tensile strains to NNO films \\ using FE/ferroelastic domain switching of FE substrates. An unprecedented electric-field-induced large \\ out-of-plane compressive strain $(-0.53 \%)$ and in-plane tensile strain $(+0.81 \%)$ are achieved in the 25 -nm \\ NNO film by switching the polarization direction of the PIN-PMN-PT substrate at T $=200 \mathrm{~K}$. This value is \\ approximately 7.4 to 45 times larger than those previously reported in FE substrate-based \\ heterostructures. As a result of the induced large lattice strain, the resistivity of the NNO film is modulated \\ up to $125 \%$. Further, taking advantage of the linear piezoelectric strain, a quantitative relationship between \\ the resistivity and the in-plane strain of the NNO film is established, with a gauge fact of $(\Delta \rho /$ \\ $\rho) / \delta \in X X \sim 40.8$. Moreover, using the domain-engineered FE/ferroelastic switching of PMN-PT substrates, \\ multiple stable resistance states with good retention and endurance properties can be obtained at room \\ temperature and the metal-to-insulator transition temperature ( $\mathrm{T} \mathrm{MI}$ ) of NNO films can be modified by \\ precisely controlling the electric-field-pulse sequence as a result of the nonvolatile remnant strain \\ transferring from the PMN-PT to the NNO film. Our results demonstrate that the electric-field-tunable \\ ferroelastic/piezoelectric strain approach can be utilized to gain deeper insight into the intrinsic strain- \\ property relationship of perovskite nickelate films and provide a simple and energy efficient way to \\ construct multistate resistive memories. \\ Disciplines \\ Engineering | Physical Sciences and Mathematics

\section{Publication Details} \\ Yan, J., Xu, M., Chen, T., Yang, M., Liu, F., Wang, H., Guo, L., Xu, Z., Fan, F., Gao, G., Dong, S., Li, X., Luo, H., \\ Zhao, W. \& Zheng, R. (2019). Manipulation of the Electronic Transport Properties of Charge-Transfer Oxide \\ Thin Films of NdNi O3 Using Static and Electric-Field-Controllable Dynamic Lattice Strain. Physical Review \\ Applied, 11 (3), 034037-1-034037-15.
}

\section{Authors}

Jian-Min Yan, Meng Xu, Ting-Wei Chen, Ming Yang, Fei Liu, Hui Wang, Lei Guo, Zhi-Xue Xu, Fang-Yuan Fan, Guan-Yin Gao, Si-Ning Dong, Xiao-Guang Li, Hao Luo, Weiyao Zhao, and Ren-Kui Zheng 


\title{
Manipulation of the Electronic Transport Properties of Charge-Transfer Oxide Thin Films of $\mathrm{NdNiO}_{3}$ Using Static and Electric-Field-Controllable Dynamic Lattice Strain
}

\author{
Jian-Min Yan, ${ }^{1}$ Meng Xu, ${ }^{1}$ Ting-Wei Chen, ${ }^{2}$ Ming-Min Yang, ${ }^{3}$ Fei Liu, ${ }^{1}$ Hui Wang, ${ }^{2}$ Lei Guo, ${ }^{1}$ \\ Zhi-Xue Xu, ${ }^{1}$ Fang-Yuan Fan, ${ }^{1}$ Guan-Yin Gao, ${ }^{4}$ Si-Ning Dong, ${ }^{5,}{ }^{*}$ Xiao-Guang Li, ${ }^{4}$ Hao-Su Luo, ${ }^{1}$ \\ Weiyao Zhao, ${ }^{6, \dagger}$ and Ren-Kui Zheng ${ }^{1,2, *}$ \\ ${ }^{1}$ State Key Laboratory of High Performance Ceramics and Superfine Microstructure, Shanghai Institute of \\ Ceramics, Chinese Academy of Sciences, Shanghai 200050, China \\ ${ }^{2}$ School of Materials Science and Engineering, Nanchang University, Nanchang 330031, China \\ ${ }^{3}$ Department of Physics, University of Warwick, Coventry CV4 7AL, United Kingdom \\ ${ }^{4}$ Hefei National Laboratory for Physical Sciences at the Microscale, Department of Physics, and Collaborative \\ Innovation Center of Advanced Microstructures, University of Science and Technology of China, \\ Hefei 230026, China \\ ${ }^{5}$ Department of Physics, University of Notre Dame, Indiana 46556, USA \\ ${ }^{6}$ ISEM, Innovation Campus, University of Wollongong, Wollongong, NSW 2500, Australia
}

(Received 27 September 2018; revised manuscript received 5 January 2019; published 15 March 2019)

\begin{abstract}
Using perovskite-type charge-transfer oxide thin films of $\mathrm{NdNiO}_{3}$ (NNO) as a model system, we demonstrate that the effects of lattice strain on the electronic transport properties can be more comprehensively understood by growing NNO films on a number of (001)-, (011)-, and (111)-cut single-crystal substrates with different lattice mismatches including the relaxor-based $0.31 \mathrm{~Pb}\left(\operatorname{In}_{1 / 2} \mathrm{Nb}_{1 / 2}\right)$ $\mathrm{O}_{3}-0.35 \mathrm{~Pb}\left(\mathrm{Mg}_{1 / 3} \mathrm{Nb}_{2 / 3}\right) \mathrm{O}_{3}-0.34 \mathrm{PbTiO}_{3}(\mathrm{PIN}-\mathrm{PMN}-\mathrm{PT})$ and $0.71 \mathrm{~Pb}\left(\mathrm{Mg}_{1 / 3} \mathrm{Nb}_{2 / 3}\right) \mathrm{O}_{3}-0.29 \mathrm{PbTiO}_{3}(\mathrm{PMN}-$ $\mathrm{PT}$ ) ferroelectric (FE) single crystals. In addition to the static lattice strains from conventional substrates (e.g., $\mathrm{SrTiO}_{3}, \mathrm{LaAlO}_{3}$ ), we in situ impose in-plane compressive or tensile strains to NNO films using $\mathrm{FE} /$ ferroelastic domain switching of FE substrates. An unprecedented electric-field-induced large out-ofplane compressive strain $(-0.53 \%)$ and in-plane tensile strain $(+0.81 \%)$ are achieved in the $25-\mathrm{nm}$ NNO film by switching the polarization direction of the PIN-PMN-PT substrate at $T=200 \mathrm{~K}$. This value is approximately 7.4 to 45 times larger than those previously reported in FE substrate-based heterostructures. As a result of the induced large lattice strain, the resistivity of the NNO film is modulated up to $125 \%$. Further, taking advantage of the linear piezoelectric strain, a quantitative relationship between the resistivity and the in-plane strain of the NNO film is established, with a gauge fact of $(\Delta \rho / \rho) / \delta \varepsilon_{x x} \sim$ 40.8. Moreover, using the domain-engineered FE/ferroelastic switching of PMN-PT substrates, multiple stable resistance states with good retention and endurance properties can be obtained at room temperature and the metal-to-insulator transition temperature $\left(T_{\mathrm{MI}}\right)$ of NNO films can be modified by precisely controlling the electric-field-pulse sequence as a result of the nonvolatile remnant strain transferring from the PMN-PT to the NNO film. Our results demonstrate that the electric-field-tunable ferroelastic/piezoelectric strain approach can be utilized to gain deeper insight into the intrinsic strain-property relationship of perovskite nickelate films and provide a simple and energy efficient way to construct multistate resistive memories.
\end{abstract}

DOI: 10.1103/PhysRevApplied.11.034037

\section{INTRODUCTION}

Strongly correlated perovskite-type $3 d$ transition-metal oxides have received considerable attention in the past several decades because of their intriguing physical

\footnotetext{
*dongsn@ustc.edu.cn

†wz929@uowmail.edu.au

†zrk@ustc.edu
}

properties, such as superconductivity [1], multiferroicity [2], metal-to-insulator transition [3], charge, spin, and orbital orderings [4,5], and colossal magnetoresistance [6]. Nickelates of $R \mathrm{NiO}_{3}(R=$ rare-earth ions and $R \neq \mathrm{La})$ is one of such systems that undergoes sharp metal-toinsulator transitions as the transition temperature $\left(T_{\mathrm{MI}}\right)$ decreases with increasing ionic radius of rare-earth ions. The low-temperature insulating nickelates are generally regarded as charge-transfer gap insulators with an empty 
Ni $3 d$ band and an occupied O $2 p$ band [4]. A recent report showed that the metal-to-insulator transition in nickelates arises from the charge disproportionation in the $\mathrm{Ni}$ sites $\left(\mathrm{Ni}^{3+} \rightarrow \mathrm{Ni}^{+\delta}+\mathrm{Ni}^{3-\delta}\right)$, which is accompanied by an orthorhombic $(\mathrm{Pbnm})$ to monoclinic $\left(P 2_{1} / n\right)$ structural phase transition [5]. Furthermore, the metal-to-insulator transition can be manipulated by a variety of methods, such as chemical doping [7], hydrostatic pressure [8,9], electrostatic doping [10], epitaxial strain [11], and so on.

$\mathrm{NdNiO}_{3}$ (NNO) has been at the center of the research owing to its sharp first-order metal-to-insulator transition and associated structural and magnetic anomalies. It is known that epitaxial strain has significant impacts on the electronic transport properties of NNO films [11-16]. Until now, previous works mainly focused on the static lattice strain effects induced by lattice mismatch between NNO films and the underlying substrates. A number of works have demonstrated that the oxygen content also has a great influence on the valence state of $\mathrm{Ni}^{3+}$ ions and thus the resistivity, $T_{\mathrm{MI}}$, and the width of the resistivity hysteresis loops. In addition to the oxygen content, some other factors, such as the thickness of a dead layer, disorder, defects, crystallinity, and so on, also have strong influences on the electronic transport properties of $\mathrm{NNO}$ films.

Perovskite-type binary and ternary ferroelectric (FE) single crystals of $(1-x) \mathrm{Pb}\left(\mathrm{Mg}_{1 / 3} \mathrm{Nb}_{2 / 3}\right) \mathrm{O}_{3}-x \mathrm{PbTiO}_{3}$ $(\mathrm{PMN}-x \mathrm{PT})$ and $(1-x-y) \mathrm{Pb}\left(\mathrm{In}_{1 / 2} \mathrm{Nb}_{1 / 2}\right) \mathrm{O}_{3}-x \mathrm{~Pb}$ $\left(\mathrm{Mg}_{1 / 3} \mathrm{Nb}_{2 / 3}\right) \mathrm{O}_{3}-y \mathrm{PbTiO}_{3}$ (PIN- $x$ PMN- $y$ PT) exhibit excellent $\mathrm{FE}$ and piezoelectric properties [17-20] as well as large ferroelectric-domain-switching-induced lattice strains, which can be reversibly, continuously, and quantitatively tuned by simply applying dc or ac electric fields to the FE crystals along the thickness direction. As of now, finely polished (001)-, (011)-, and (111)-cut PMN$x \mathrm{PT}$ and PIN- $x \mathrm{PMN}-y \mathrm{PT}$ single crystals have been used as substrates to grow a variety of thin films, such as $R_{1-x} A_{x} \mathrm{MnO}_{3}(R=\mathrm{La}, \mathrm{Pr}, A=\mathrm{Ca}, \mathrm{Sr}, \mathrm{Ba})$ [21-29], Co [30,31], $\mathrm{BiFeO}_{3}$ [32], $\mathrm{YBa}_{2} \mathrm{Cu}_{3} \mathrm{O}_{7}$ [33,34], $\mathrm{BaTiO}_{3}: \mathrm{Yb} / \mathrm{Er}$ $[35,36], A \mathrm{Fe}_{2} \mathrm{O}_{4}(A=\mathrm{Co}, \mathrm{Ni})[37,38], \mathrm{Fe}_{3} \mathrm{O}_{4}[39,40]$, and $\mathrm{Bi}_{0.94} \mathrm{~Pb}_{0.06} \mathrm{CuSeO}$ [41], so that the lattice strain and the related properties of these films could be in situ modified. Virtually, using this unique method, the intrinsic lattice strain effects of any films grown on FE substrates can be studied without introducing extrinsic effects caused by the variation in oxygen content, thickness of the dead layer, defects, crystallinity, disorder, and so on. For instance, Jang et al. [42] found that the metal-to-insulator transition temperature of NNO film can be shifted to a lower temperature by approximately $3.3 \mathrm{~K}$ in response to the $0.25 \%$ in-plane compressive strain by applying a $+10 \mathrm{kV} / \mathrm{cm}$ electric field to the $\mathrm{NNO} / \mathrm{SrTiO}_{3}(\mathrm{STO}) / \mathrm{PMN}-x \mathrm{PT}$ structures, demonstrating that electric-field-controllable strain engineering is a useful tool to tune the electronic properties of NNO films.
To obtain a more comprehensive understanding of the lattice strain effects in NNO films, in this paper, we grow NNO films on (001)-oriented conventional single-crystal substrates including $\mathrm{YAlO}_{3}(\mathrm{YAO}), \mathrm{SrLaAlO}_{4}(\mathrm{SLAO})$, $\mathrm{LaAlO}_{3}(\mathrm{LAO}),\left(\mathrm{LaAlO}_{3}\right)_{0.3}\left(\mathrm{Sr}_{2} \mathrm{AlTaO}_{6}\right)_{0.7}(\mathrm{LSAT}), \mathrm{SrTiO}_{3}$ (STO), $\mathrm{KTaO}_{3}(\mathrm{KTO})$, and piezoelectrically active (001)oriented $\quad 0.31 \mathrm{~Pb}\left(\mathrm{In}_{1 / 2} \mathrm{Nb}_{1 / 2}\right) \mathrm{O}_{3}-0.35 \mathrm{~Pb}\left(\mathrm{Mg}_{1 / 3} \mathrm{Nb}_{2 / 3}\right) \mathrm{O}_{3}$ $-0.34 \mathrm{PbTiO}_{3}$ (PIN-PMN-PT), and (011)- and (111)oriented $0.71 \mathrm{~Pb}\left(\mathrm{Mg}_{1 / 3} \mathrm{Nb}_{2 / 3}\right) \mathrm{O}_{3}-0.29 \mathrm{PbTiO}_{3}$ (PMN-PT) single-crystal substrates. We in situ manipulate the inplane strain of NNO films through electric-field-induced FE/ferroelastic-domain switching and the converse piezoelectric effect of the PIN-PMN-PT and PMN-PT single crystals and find a ferroelastic strain-driven large increase in the resistivity up to approximately $125 \%$. Using the piezoelectric strain of PIN-PMN-PT, we establish a quantitative relationship between the resistivity and the inplane strain and obtain Possion's ratio of NNO films. Our findings demonstrate that a significantly large inplane tensile strain up to $+0.81 \%$ can be induced in situ using the ferroelastic effect of ternary PIN-PMNPT single-crystal substrates. Further, taking advantage of the domain-engineered $\mathrm{FE} /$ ferroelastic switching of a PMN-PT substrate, multiple stable resistance states and modulation of $T_{\mathrm{MI}}$ can be achieved using electric fields.

\section{EXPERIMENTAL DETAILS}

PIN-PMN-PT and PMN-PT single-crystal boules with a size of $\varnothing 50 \mathrm{~mm} \times 80 \mathrm{~mm}$ are grown by the modified Bridgman technique at the Shanghai Institute of Ceramics, Chinese Academy of Sciences. The as-grown crystal boules are cut into small pieces with a size of $5 \times 5 \times 0.5 \mathrm{~mm}^{3}$ and carefully polished to a root-mean-square roughness $R_{\mathrm{rms}} \sim 1.2 \mathrm{~nm}$. Such polished PIN-PMN-PT(001), PMNPT(011), and PMN-PT(111) single-crystal substrates show good FE and piezoelectric properties. Figures $1(\mathrm{e})$ and $1(\mathrm{~g})$ show a representative polarization-electric-field hysteresis loop and a butterfly-shaped strain-electric-field loop for the PIN-PMN-PT(001) substrate, respectively. NNO films are grown on one-side polished and (001)-oriented YAO, SLAO, LAO, LSAT, STO, KTO, PIN-PMN-PT, and (011)and (111)-oriented PMN-PT single-crystal substrates by pulsed laser deposition (PLD) using a $\mathrm{KrF}$ excimer laser $(\lambda=248 \mathrm{~nm})$. During film deposition, the oxygen pressure, laser energy density, pulse repetition rate, and substrate temperature are kept at $30 \mathrm{~Pa}, 2 \mathrm{~J} / \mathrm{cm}^{2}, 5 \mathrm{~Hz}$, and $700{ }^{\circ} \mathrm{C}$, respectively. The distance between the NNO target and the substrates is $6 \mathrm{~cm}$. The as-grown NNO films are in situ annealed in $1 \mathrm{~atm}$ oxygen for $60 \mathrm{~min}$ before cooling down to room temperature at a rate of $5{ }^{\circ} \mathrm{C} / \mathrm{min}$.

The phase purity, crystallinity, and epitaxial properties are characterized by x-ray diffraction (XRD) $\theta-2 \theta, \omega$, and $\phi$ scans as well as the reciprocal space map (RSM) using a PANalytical X'Pert PRO x-ray diffractometer with 
(a)

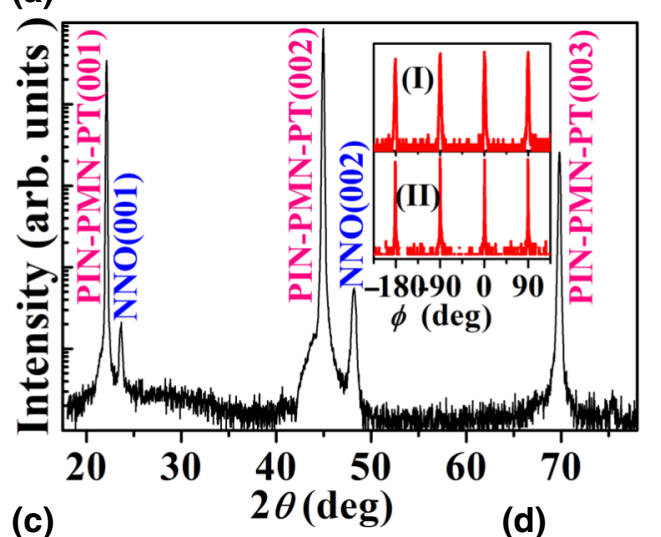

(c)

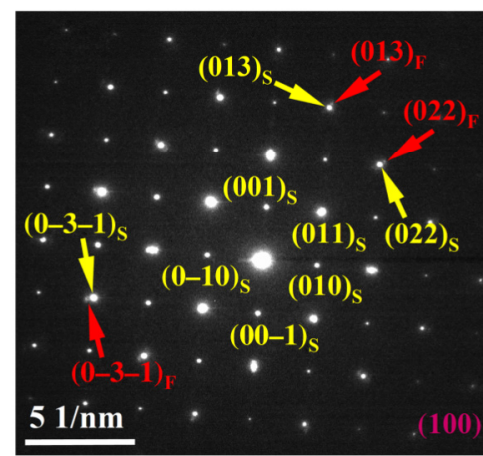

(b)

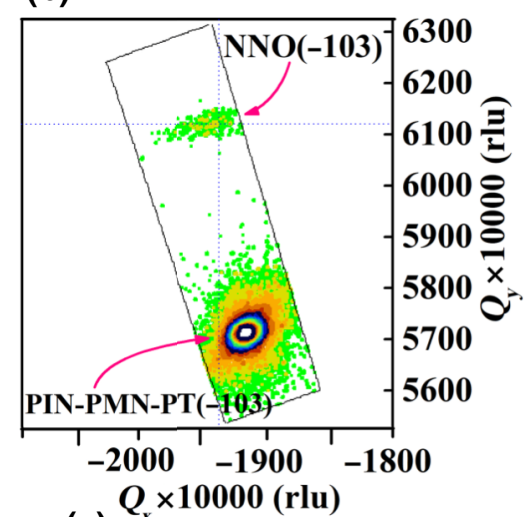

FIG. 1. (a) XRD $\theta-2 \theta$ scan pattern for the $\mathrm{NNO}(25 \mathrm{~nm}) / \mathrm{PIN}-\mathrm{PMN}$ $\mathrm{PT}(001)$ structure. Insets (I) and (II): $\phi$ scan patterns taken on the NNO (101) and PIN-PMN-PT (101) diffraction peaks, respectively. (b) Reciprocal space mapping of the NNO film in the vicinity of the PIN-PMNPT and NNO (-103) reflections. (c) SAED pattern taken at the interface for the NNO/PIN-PMN-PT structure. Yellow and red arrows indicate the diffraction patterns from the PINPMN-PT and the NNO film, respectively. (d) A box-in-box piezoresponse force microscopy image of the PINPMN-PT under positively polarized (inner box) and negatively polarized (outer box) states. (e) Polarizationelectric-field hysteresis loop for the PINPMN-PT substrate. (f) Cross-section SEM image of the NNO/PIN-PMN-PT structure. (g) In-plane strain vs electric field for the PIN-PMN-PT substrate.

$\mathrm{CuK}_{\alpha 1}$ radiation $(\lambda=1.5406 \AA)$. A cross-section scanning electron microscopy (SEM) is measured using a JSM$6700 \mathrm{~F}$ scanning electron microscope. The selected area electron diffraction (SAED) is measured using a Tecnai G2F20 S-Twin transmission electron microscope. The FE properties of the PIN-PMN-PT(001), PMN-PT(011), and PMN-PT(111) substrates at microscale $\left(5 \times 5 \mu \mathrm{m}^{2}\right)$ are characterized by piezoresponse force microscopy (PFM) using a Dimension V (Veeco) scanning probe microscope.

The resistivity of NNO films is measured using a physical property measurement system (PPMS-9, Quantum Design). The initial poling of PIN-PMN-PT and PMN-PT substrates and the followed polarization direction rotation are achieved by applying dc electric fields $(E)$ to PIN-PMN-PT and PMN-PT substrates along the thickness direction using the conducting NNO film (approximately $25 \mathrm{~nm}$ ) and the $\mathrm{Ag}$ film (approximately $100 \mathrm{~nm}$ ) as the top and bottom electrodes, respectively. Schematic of the experimental setups for in situ measurements of resistivity using the four-probe technique and electricfield-induced out-of-plane and in-plane strains in the NNO films and PIN-PMN-PT and PMN-PT substrates using the XRD are shown in Ref. [43]. The initial FE-poling and $180^{\circ}$ and non- $180^{\circ}$ polarization-rotation-induced in-plane strains in PIN-PMN-PT and PMN-PT substrates are measured using a strain gauge that is attached to the surface of the substrates with epoxy. The polarization-electric field $(P-E)$ hysteresis loops of the PIN-PMN-PT and PMN-PT substrates are measured at room temperature using a Precision Multiferroic Analyzer (Radiant Technologies, Inc., USA).

\section{RESULTS AND DISCUSSION}

Figure 1(a) shows the out-of-plane XRD $\theta-2 \theta$ scan pattern for the $25-\mathrm{nm}$ NNO film grown on a PIN-PMN-PT substrate. Only $(00 l)(l=1,2)$ diffraction peaks from the NNO film and PIN-PMN-PT substrate appear, indicating that the NNO film is single phase and highly (001)oriented. XRD $\phi$ scans taken on the NNO (101) and PIN-PMN-PT (101) diffraction peaks show that four-fold symmetrical diffraction peaks from the NNO film appear every $90^{\circ}$, occurring at the same azimuthal angles as those of PIN-PMN-PT substrate [Insets (I) and (II) of Fig. 1(a)], a sign of epitaxial growth of the NNO film on the PINPMN-PT substrate. RSM is conducted to investigate the coherent growth of the NNO film. Figure 1(b) shows the RSM pattern taken around the $(-103)_{\mathrm{PC}}$ diffraction peak of the NNO film and PIN-PMN-PT substrate. The results reveal that the in-plane lattice of the NNO film shows slight relaxation, as reflected by the slight shift of the NNO (-103) diffraction spot along the $Q_{x}$ axis. Figure 1(c) shows a SAED pattern taken from both the NNO film and the PIN-PMN-PT substrate. Due to the quite large lattice mismatch between the NNO film and the PIN-PMN-PT substrate, two appreciable sets of diffraction patterns can 
be identified, corresponding to the PIN-PMN-PT (strong spots) and the NNO film (weak spots) lattices, respectively. This crystallographic orientation relationship between the NNO film and the PIN-PMN-PT substrate is consistent with the XRD analysis, which further confirms the epitaxial growth of the NNO film on the PIN-PMN-PT(001) substrate. An atomic force microscopy (AFM) image reveals that the NNO film has a flat surface with $R_{\mathrm{rms}}=1.5 \mathrm{~nm}$ (see Ref. [43]). A cross-section SEM image shows that the thickness of the NNO film is approximately $25 \mathrm{~nm}$. The above characterizations show that the NNO film has a good structural quality. We note that the NNO films grown on the YAO, SLAO, LAO, LSAT, STO, and KTO singlecrystal substrates also show good in-plane and out-of-plane alignments with respect to the corresponding substrates.

Utilizing the aforementioned single-crystal substrates with different lattice constants, the NNO epitaxial films are expected to be subjected to in-plane compressive to tensile strain with increasing lattice constants of the substrates. Figure 2(a) shows the lattice mismatch between the NNO bulk and the substrates used in this study. The XRD out-of-plane $\theta-2 \theta$ scans show that the NNO (002) diffraction peaks, as indicated by the arrows in Fig. 2(c), systematically shift to lower $2 \theta$ angles with increasing inplane compressive strain from the substrates. Probably due to the smallest lattice mismatch between the NNO and the LAO, a finite size oscillation of the XRD pattern is observed for the NNO/LAO thin-film sample [Fig. 2(c)],
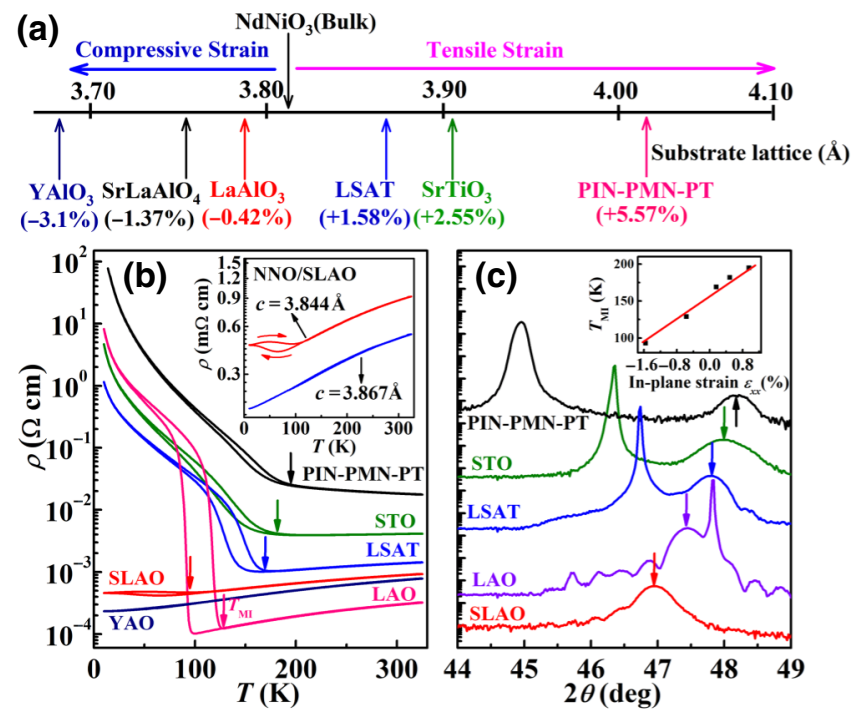

FIG. 2. (a) Lattice mismatch between the NNO and the substrates used in this study. (b) Temperature dependence of resistivity for NNO films on different substrates. Inset: Temperature dependence of resistivity for NNO films grown on SLAO substrates with different in-plane strains. (c) XRD $\theta-2 \theta$ scans around the (002) diffraction peaks for NNO films on different substrates. Inset: Metal-to-insulator transition temperature $T_{\mathrm{MI}}$ vs in-plane strain $\varepsilon_{x x}(\%)$. implying the excellent crystalline quality and flat surface of this film. The effects of the substrate-induced static strain on the resistivity are shown in Fig. 2(b), where both the metal-to-insulator transition temperature $\left(T_{\mathrm{MI}}\right)$ and the transition width $(\Delta T)$ between the cooling and heating cycles are strongly modified by the epitaxial strain. Among all the NNO films, the NNO film on the LAO substrate exhibits the most dramatic resistivity increase (approximately five orders in magnitude) below $T_{\mathrm{MI}}$ and shows the largest transition width, probably due to its good crystalline quality and lowest in-plane lattice mismatch between the film and the LAO substrate. In contrast, the NNO film grown on the SLAO substrate is subjected to an out-of-plane tensile strain $\left(\varepsilon_{\mathrm{zz}}=1.0 \%\right)$ and an in-plane compressive strain $\left(\varepsilon_{x x}=-0.66 \%\right)$, and thus shows a very stabilized metallic state and marginal metal-to-insulator transition. A re-entrant-like metallic state and a small resistivity hysteresis between cooling and heating cycles appear between $T=20 \mathrm{~K}$ and $T=106 \mathrm{~K}$ for this sample [inset of Fig. 2(b)], which are similar to those observed in the $\mathrm{NNO}(200 \mathrm{~nm}) / \mathrm{LAO}(001)$ thin-film sample [13]. These phenomena are due to the melting of the insulating state under a large in-plane compressive strain, which closes the charge-transfer gap [14]. We prepare another NNO/SLAO thin-film sample with a larger in-plane compressive strain $\left(\varepsilon_{x x}=-1.06 \%\right)$ and find that its metal-to-insulator transition is completely suppressed and the film is metallic down to the lowest temperature [See the blue curve in the inset of Fig. 2(b).]. In contrast, with increasing in-plane tensile strain, both $T_{\mathrm{MI}}$ and resistivity at $T=300 \mathrm{~K}$ increase significantly [Fig. 2(b)]. To derive the dependence of $T_{\mathrm{MI}}$ on the static strain, we set $T_{\mathrm{MI}}$ as the temperature where the transition hysteresis begins. A series of $T_{\mathrm{MI}}$ and their dependence on the in-plane strain can be determined. One can find that $T_{\mathrm{MI}}$ decreases approximately linearly with the in-plane compressive strain [inset of Fig. 2(c)].

\section{A. NNO/PIN-PMN-PT(001)}

In order to further probe into the effects of in-plane strain on the electronic transport properties of NNO films, we in situ modify the strain state of the $25-\mathrm{nm}$ NNO film by poling the PIN-PMN-PT substrate along the $\langle 001\rangle$ crystallographic direction and simultaneously measuring the relative resistivity change $(\Delta \rho / \rho)$ of the NNO film. Here, $\Delta \rho / \rho=[\rho(E)-\rho(0)] / \rho(0)$, where $\rho(E)$ and $\rho(0)$ are the resistivity of the NNO film when an electric field $E$ or a zero electric field is applied to the PIN-PMN-PT substrate, respectively. As shown in Fig. 3(a), $\Delta \rho / \rho$ is nearly field independent for $E<2.1 \mathrm{kV} / \mathrm{cm}$ and decreases significantly in the electric-field range of $2.1 \mathrm{kV} / \mathrm{cm}<E<3.4 \mathrm{kV} / \mathrm{cm}$. For $E>4 \mathrm{kV} / \mathrm{cm}$, the resistivity decreases linearly with increasing $E$, which arises from the linear increase in the in-plane compressive strain from the PIN-PMN-PT substrate [44]. This will be discussed in more detail later. 
(a)

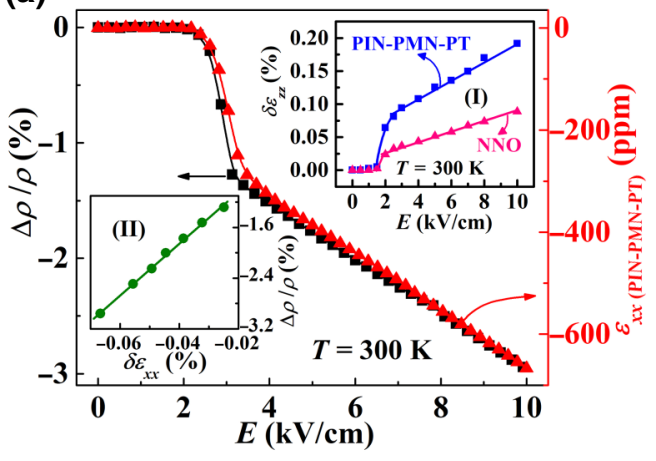

(b)

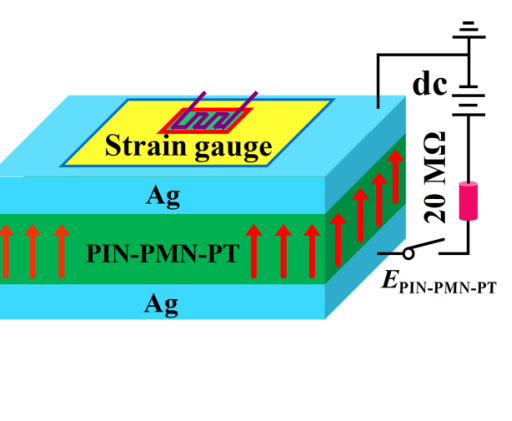

FIG. 3. Relative resistivity change $(\Delta \rho / \rho)$ of the NNO film and the electric-field-induced in-plane strain of the PIN-PMN-PT substrate as a function of the electric field $(E)$ applied to the PIN-PMN-PT substrate. Inset (I): Electric-field-induced out-of-plane strain $\left(\delta \varepsilon_{z z}\right)$ for the NNO film and the PIN-PMN-PT substrate. Inset (II): Electric-field-induced in-plane strain $\left(\delta \varepsilon_{x x}\right)$ vs $\Delta \rho / \rho$ for the NNO film. (b) Schematic of the experimental setup for the measurements of the electric-field-induced in-plane strain in the PIN-PMNPT(001) substrate.

It is noted that the variation of $\Delta \rho / \rho$ with $E$ (the black curve) tracks the electric-field-induced in-plane compressive strain $\left[\varepsilon_{x x}\right.$ (PIN-PMN-PT) $]$ of the PIN-PMN-PT substrate (the red curve) quite well, strongly indicating that it is the $71^{\circ}$ and $109^{\circ} \mathrm{FE}$ domain switching-induced lattice strain that modifies the resistivity of the NNO film. Further, we estimate the effects of the poling-induced polarization charges on the resistivity (i.e., the FE-field effect). Here, the polarization direction pointing to the NNO film is defined as positive poling, as schematically illustrated in Fig. 3(b). The poling-induced relative change in the volume carrier density $(\Delta n / n)$ can be calculated using $\Delta n / n=\Delta$ P/edn $[24,45]$, where $n$ and $d$ are the volume carrier density and thickness of the NNO film, $\Delta P$ is the polarization of the PIN-PMN-PT substrate, and $e$ is the unit charge $\left(1.602 \times 10^{-19} \mathrm{C}\right)$. Using $\Delta P \sim 25 \mu \mathrm{C} / \mathrm{cm}^{2}$ at $E=10 \mathrm{kV} / \mathrm{cm}, d \sim 25 \mathrm{~nm}$ [Fig. 1(f)], and $n=5 \times 10^{22} / \mathrm{cm}^{3}$ for the NNO film [10], one obtains $\Delta n / n \sim-0.12 \%$ for $E=10 \mathrm{kV} / \mathrm{cm}$ for the $p$ type NNO [10]. In the first-order approximation, $\Delta \rho / \rho \approx-\Delta n / n$ [45]. Therefore, if only the FE-field effect is taken into account, the resistivity of the NNO film should increase by approximately $0.12 \%$ due to the depletion of the hole-carrier density. This is sharply inconsistent with the observed decrease in the resistivity upon positive poling of the PIN-PMN-PT. Moreover, the magnitude of the relative resistivity change (approximately $0.12 \%$ ) estimated from the FE-field effect is minor.

To quantitatively understand the effects of the electricfield-induced strain on the resistivity, we measure the electric-field-induced out-of-plane strain $\left(\varepsilon_{z z}\right)$ of both the NNO film and the PIN-PMN-PT substrate during the initial poling process using XRD and show the results in inset (I) of Fig. 3(a). Here $\delta \varepsilon_{z z}=\left[\varepsilon_{z z}(E)-\varepsilon_{z z}(0)\right] / \varepsilon_{z z}(0)$, where $\varepsilon_{z z}(E)$ and $\varepsilon_{z z}(0)$ are the out-of-plane lattice strains of the NNO film and PIN-PMN-PT substrate when the PIN-PMN-PT substrate is under an electric field $E$ and

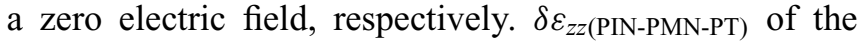
PIN-PMN-PT substrate increases significantly in the field region of $1.5 \mathrm{kV} / \mathrm{cm} \leq E \leq 2.5 \mathrm{kV} / \mathrm{cm}$ (near the coercive field of the PIN-PMN-PT) and increases linearly with a further increase in $E$ from 2.5 to $10 \mathrm{kV} / \mathrm{cm}$. The $E$ field-induced $\delta \varepsilon_{z z}$ in the PIN-PMN-PT is transferred to the NNO film, as reflected by the similar variation trend of $\delta \varepsilon_{\mathrm{zz}}$ (NNO) with $E$ for the NNO film. However, the

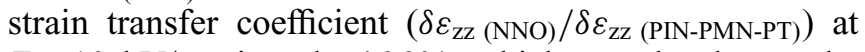
$E=10 \mathrm{kV} / \mathrm{cm}$ is only $46.3 \%$, which may be due to the lattice relaxation across the interface, arising from the quite large lattice mismatch between the NNO and the PIN-PMN-PT. According to the $\Delta \rho / \rho$ and $\delta \varepsilon_{z z(\mathrm{NNO})}$ data [Fig. 3(a)] and the Poisson ratio $v=0.43$ (See the following section for the calculation of the Poisson ratio.) for the NNO film, a quantitative relationship between $\Delta \rho / \rho$ and the in-plane strain $\delta \varepsilon_{x x(\mathrm{NNO})}$ is obtained and shown in inset (II) of Fig. 3(a). $\Delta \rho / \rho$ increases linearly with increasing in-plane compressive strain and can be expressed as $\Delta \rho / \rho=40.8 \delta \varepsilon_{x x \text { (Film) }}-0.22$.

Taking advantage of the electric-field-tunable strain in the PIN-PMN-PT substrate, we are able to obtain the effects of in-plane strain on the resistivity near $T_{\mathrm{MI}}$. We in situ modify the in-plane strain of the NNO film at $T=200 \mathrm{~K}$ (close to $T_{\mathrm{MI}}$ ) by switching the polarization from the upward direction (i.e., pointing to the NNO film, defined as the $P_{r}^{+}$state) toward the in-plane direction (i.e., approximately parallel to the film plane, defined as the $P_{r}^{\|}$ state) [see the schematic diagram shown in Fig. 4(b)]. This can be achieved by applying negative electric fields with appropriate magnitude to the positively poled PIN-PMNPT substrate. During the polarization direction rotation, the resistivity is simultaneously measured and plotted as a function of $E$ in the inset of Fig. 4(a). The $(\Delta \rho / \rho)$ vs $E$ curve exhibits a semi-square-like shape. Specifically, with increasing $E$ from 0 to $-6.6 \mathrm{kV} / \mathrm{cm}, \Delta \rho / \rho$ increases 
(a)

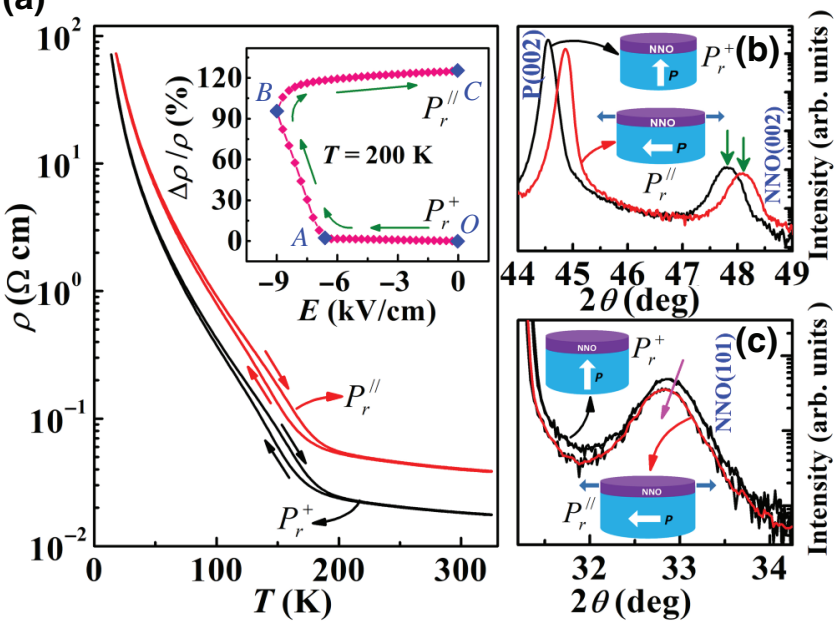

FIG. 4. (a) Temperature dependence of resistivity for the NNO film when the polarization of the PIN-PMN-PT substrate points to the NNO film and parallel to the film plane, respectively. Inset: $\Delta \rho / \rho$ of the NNO film as a function of negative electric fields applied to the positively poled PIN-PMN-PT substrate at $T=200 \mathrm{~K}$. (b),(c) XRD out-of-plane and in-plane $\theta-2 \theta$ scans around the (002) and (101) reflections when the polarization state of the PIN-PMN-PT substrate is in the $P_{r}^{+}$and $P_{r}^{\|}$states, respectively. Inset: Schematic diagrams for the polarizationrotation-induced in-plane strain in the NNO film.

slightly (approximately 2.2\%) and linearly (i.e., $\Delta \rho / \rho \propto$ $E)$, which can be ascribed to the electric-field-induced inplane tensile strain via the converse piezoelectric effect of the PIN-PMN-PT substrate [21]. Interestingly, $\Delta \rho / \rho$ increases sharply with a further increase in $E . \Delta \rho / \rho$ reaches approximately $95 \%$ at $E=-9 \mathrm{kV} / \mathrm{cm}$, which is 31 times larger than that at $T=300 \mathrm{~K}$. With decreasing $E$ from -9 to $0 \mathrm{kV} / \mathrm{cm}, \Delta \rho / \rho$ increases slightly and linearly to approximately $125 \%$. Due to the $p$ type nature of the NNO film $[10,46]$, if the polarization state of the PIN-PMN-PT is switched from positive [corresponding to the polarization state of point $O$ in the inset of Fig. 4(a)] to negative (corresponding to the polarization state of point $C$ ), the hole-carrier density of the $\mathrm{NNO}$ film will increase due to the electric-field-induced positive polarization charges at the interface between the NNO film and the PIN-PMN-PT substrate. Consequently, the resistivity is expected to decrease, which, however, is completely inconsistent with the large increase in $\Delta \rho / \rho$ by approximately $125 \%$ associated with the polarization switching. Therefore, the polarization-switching-induced interfacial charge effect fails to explain the electric-fieldinduced large resistivity change. It is also noted that a similar phenomenon can be achieved at $T=200 \mathrm{~K}$ for the NNO/PMN-PT(001) heterostructure (see Fig. 5).

A plausible explanation is still the polarizationswitching-induced strain that has been transferred to the NNO film, thereby inducing a large in-plane tensile strain

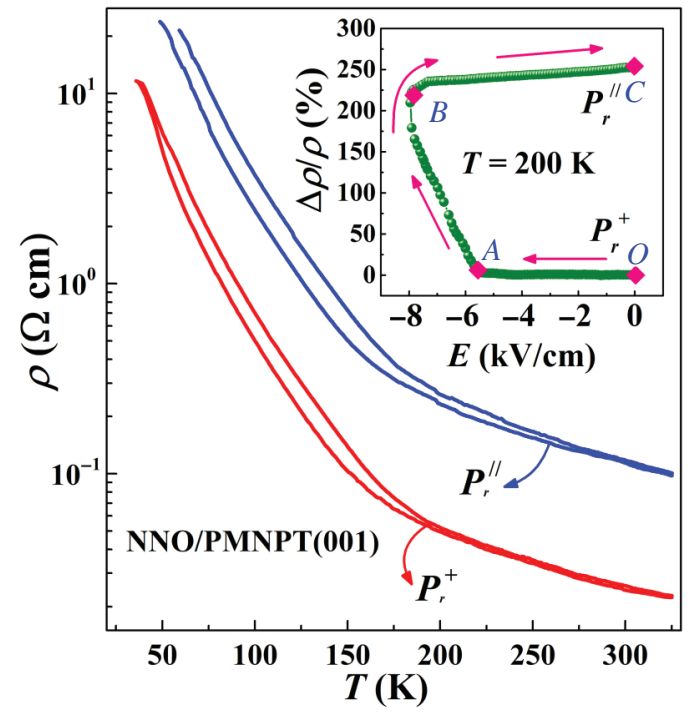

FIG. 5. Temperature dependence of resistivity for the NNO film when the polarization of the PMN-PT(001) substrate points to the NNO film and parallel to the film plane, respectively. Inset: $\Delta \rho / \rho$ of the NNO film as a function of negative electric fields applied to the positively poled PMN-PT(001) substrate at $T=200 \mathrm{~K}$.

in the NNO film. With decreasing temperature from 300 to $200 \mathrm{~K}$, the PIN-PMN-PT substrate enters into a nonergodic (glassy) state where the correlation among electric dipoles becomes stronger, resulting in inhomogeneous distribution of coercive fields and thus an enhanced average coercive field [47]. Consequently, applying a negative electric field to the positively poled PIN-PMN-PT will induce incomplete switching of positive aligned electric dipoles, thus causing an expansion of the in-plane lattice and a contraction of the out-of-plane lattice [47] of the PIN-PMN-PT substrate.

To confirm this, both out-of-plane and in-plane XRD $\theta-2 \theta$ scans are performed on the NNO/PIN-PMN-PT sample at room temperature after applying a negative electric field to the positively poled PIN-PMN-PT substrate at $T=200 \mathrm{~K}$. As shown in Fig. 4(b), both the PIN-PMNPT (002) and NNO (002) diffraction peaks significantly shift toward higher $2 \theta$ values, indicating that the out-ofplane lattice constant $c$ for the $P_{r}^{\|}$state [corresponding to the strain state of point $C$ in the inset of Fig. 4(a)] is smaller than that for the $P_{r}^{+}$state [corresponding to the strain state of point $O$ in the inset of Fig. 4(a)]. The electric-field-induced out-of-plane compressive strain $\delta \varepsilon_{z z}$ is calculated to be $-0.53 \%$ for the NNO film. Here, $\delta \varepsilon_{z z}$ is defined as $\delta \varepsilon_{z z}=\left(c_{P_{r}^{\|}}-c_{P_{r}^{+}}\right) / c_{P_{r}^{+}}$where $c_{P_{r}^{\|}}$and $c_{P_{r}^{+}}$ are the $c$ axis lattice constants of the NNO film for the $P_{r}^{\|}$and $P_{r}^{+}$states, respectively. Because of the shrinkage of the out-of-plane lattice constant, the in-plane lattice constant increases accordingly due to the Poisson effect, which is directly reflected by the shift of the NNO (101) 
diffraction peaks toward a smaller $2 \theta$ value [Fig. 4(c)]. The electric-field-induced in-plane tensile strain $\delta \varepsilon_{x x}$ is calculated to be approximately $+0.81 \%$ for the NNO film. Here, $\delta \varepsilon_{x x}$ is defined as $\delta \varepsilon_{x x}=\left(a_{P_{r}^{\|}}-a_{P_{r}^{+}}\right) / a_{P_{r}^{+}}$ where $a_{P_{r}^{\|}}$and $a_{P_{r}^{+}}$are the in-plane $a$ axis lattice constants of the NNO film for the $P_{r}^{\|}$and $P_{r}^{+}$states, respectively. Such a large electric-field-induced in-plane tensile strain is approximately 7.4 to 45 times larger than those reported in other perovskite film/PMN-PT heterostructures such as the $\mathrm{La}_{0.5} \mathrm{Ca}_{0.5} \mathrm{MnO}_{3} / \mathrm{PIN}-\mathrm{PMN}-$ PT(111) [21], $\mathrm{La}_{0.6} \mathrm{Sr}_{0.4} \mathrm{MnO}_{3} / \mathrm{PMN}-\mathrm{PT}(011)$ [29], and $\mathrm{Pr}_{0.7}\left(\mathrm{Ca}_{0.6} \mathrm{Sr}_{0.4}\right)_{0.3} \mathrm{MnO}_{3} / \mathrm{PMN}-\mathrm{PT}(011)$ [48]. According to these electric-field-induced out-of-plane and in-plane strains, the Possion ratio of the NNO film is calculated to be 0.43 using $\delta \varepsilon_{z z}=-2 v /(1-v) \delta \varepsilon_{x x}$ [49]. Therefore, these in situ XRD results not only give robust evidence that the incomplete polarization switching at low temperatures induces a significantly large in-plane tensile strain in the NNO film, but also allows one to determine the Poisson ratio of the NNO film. At temperatures close to the metalto-insulator transition, the overlap between the Ni $3 d$ band and the $\mathrm{O} 2 p$ band starts to decrease and the charge transfer gap opens, leading to the charge-ordered insulating state [50-52]. Near this critical temperature, the incomplete polarization-switching-induced in-plane tensile strain will narrow the bandwidth and facilitate the growth of the charge-ordered insulating state and thus significantly enhance the resistivity as shown in Fig. 4(a). The resistivity for the $P_{r}^{\|}$state is much larger than that for the $P_{r}^{+}$state in the temperature region from $10-325 \mathrm{~K}$, suggesting that the polarization-switching-induced strain is stable up to $325 \mathrm{~K}$.

Utilizing the converse piezoelectric effect of the PIN-PMN-PT, the resistivity of the NNO film can also be dynamically manipulated. Figure 6(a) shows the relative resistivity change $(\Delta \rho / \rho)$ as a function of the electric fields applied to the positively polarized PIN-PMN-PT at different fixed temperatures from 90 to $380 \mathrm{~K}$. For $90 \mathrm{~K} \leq T \leq 350 \mathrm{~K}, \Delta \rho / \rho$ decreases linearly with an increasing electric field, which is a typical behavior of resistivity perovskite transition-metal-oxide films modulated by the converse piezoelectric effect of underlying FE substrates $[21,23,53]$, and arises from the increase in the electronic bandwidth due to the reduction of its inplane tensile strain [53]. For higher temperatures (e.g., $T=370$ and $380 \mathrm{~K}), \Delta \rho / \rho$ shows sharply changed points near $E=5$ and $2.5 \mathrm{kV} / \mathrm{cm}$ for $T=370$ and $380 \mathrm{~K}$, [the inset of Fig. 6(a)], respectively, which are probably associated with the rhombohedral-to-tetragonal structural phase transition at $T=376 \mathrm{~K}$ in the PINPMN-PT substrate (see Ref. [43]) [19,21]. Figure 6(b) shows the electric-field-induced variation in the resistivity $\left(\Delta \rho / \rho_{E}\right)$ as a function of temperature. Here, $\Delta \rho / \rho_{E}$ is calculated using $\Delta \rho / \rho_{E}=\left(\rho_{E=6.75 \mathrm{kV} / \mathrm{cm}}-\right.$ (a)

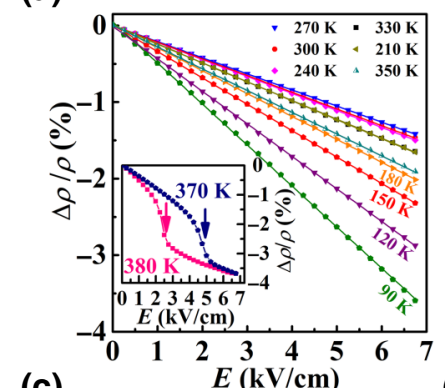

(c)

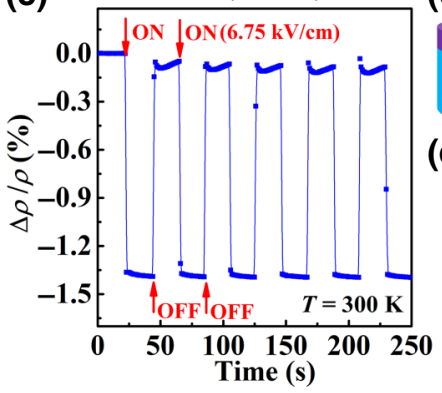

(b)

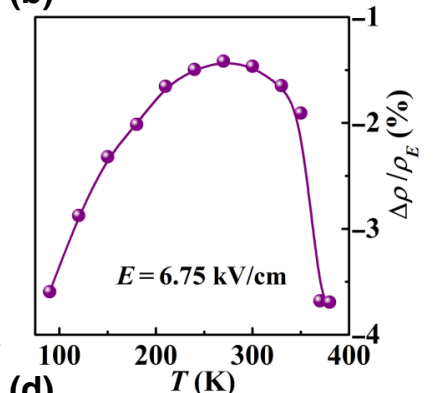

(d)

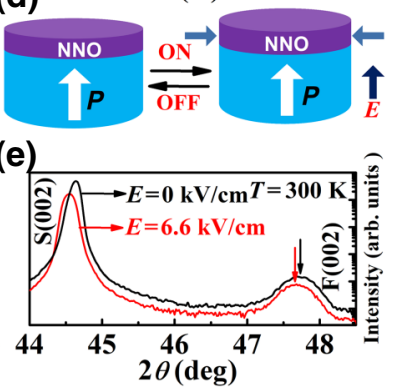

FIG. 6. (a) $\Delta \rho / \rho$ of the NNO film as a function of positive electric field $(E)$ applied to positively poled PIN-PMN-PT substrate at various fixed temperatures. Inset: $\Delta \rho / \rho$ vs electric field at $T=370$ and $380 \mathrm{~K}$. (b) Temperature dependence of the $6.75 \mathrm{kV} / \mathrm{cm}$-electric-field-induced $\Delta \rho / \rho_{E}$. (c) Dynamic modulation of the resistivity of the NNO film through the converse piezoelectric effect. (d) Schematic diagram of the converse piezoelectric effect. (e) XRD $\theta-2 \theta$ scans around the (002) diffraction peaks for the NNO/PIN-PMN-PT structure when $E=0$ and $6.75 \mathrm{kV} / \mathrm{cm}$ is applied to the positively poled PIN-PMN-PT substrate, respectively.

$\left.\rho_{E=0 \mathrm{kV} / \mathrm{cm}}\right) / \rho_{E=0 \mathrm{kV} / \mathrm{cm}} \cdot \Delta \rho / \rho_{E}$ increases with decreasing temperature from $T=270 \mathrm{~K}$, indicating that the resistivity in the insulating state is more sensitive to the piezostrain than that in the less insulating state. It is noted that the converse piezoelectric-effect-induced $\Delta \rho / \rho_{E}$ close to the metal-to-insulator transition temperature (e.g., $210 \mathrm{~K}$ ) (approximately $-1.65 \%$ under $6.75 \mathrm{kV} / \mathrm{cm}$ ) is much smaller than the polarization-switching-induced resistance change $(\Delta \rho / \rho=125 \%)$ near $200 \mathrm{~K}$ discussed above. The converse piezoelectric-effect-induced reduction in the inplane tensile strain will stabilize the metallic state to some extent, leading to a small decrease in the resistance. In contrast, the incomplete polarization switching at $200 \mathrm{~K}$ induces a large in-plane tensile strain, which could facilitate the growth of the charge-ordered insulating phase. This indicates that the metallic static is unstable when closing to the metal-to-insulator transition and is vulnerable to the in-plane tensile strain. Based on the linear relationship between the resistivity and the positive electric field exerted on positively polarized PIN-PMN-PT (001), dynamic and reversible manipulation of the resistivity of the NNO film is realized and is shown in Fig. 6(c). The resistivity drops sharply when a positive electric 
(a)

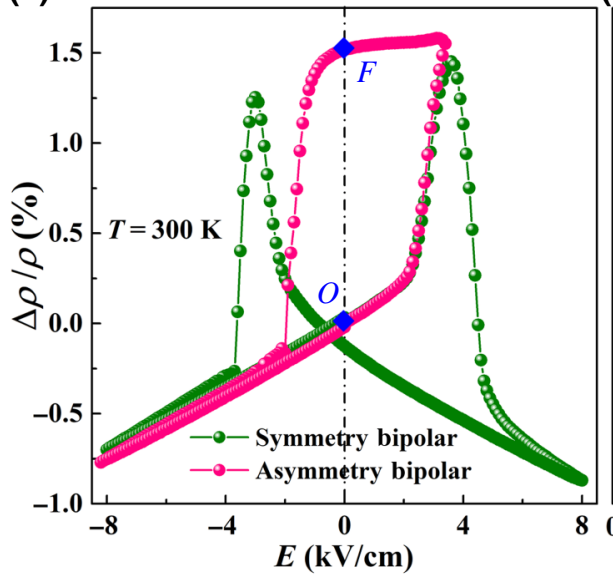

(b)

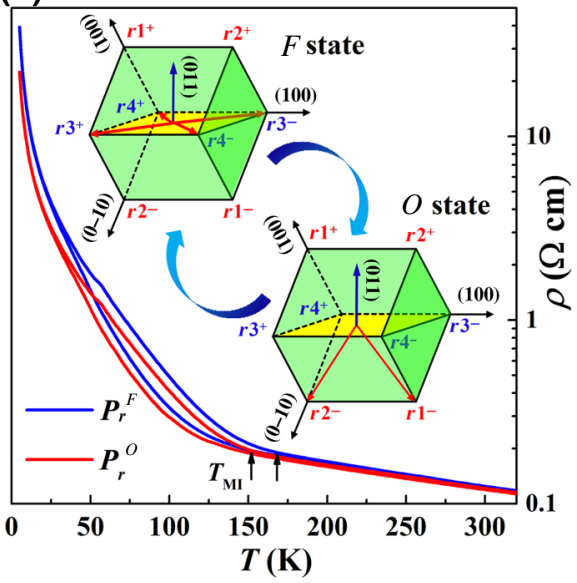

FIG. 7. (a) The relative resistivity change $(\Delta \rho / \rho)$ induced by symmetrical and asymmetrical bipolar electric-field sweeping at $T=300 \mathrm{~K}$. With the application of an asymmetrical bipolar electric field, two stable resistivity states $O$ and $F$ can be realized. (b) The resistivity of the NNO film as a function of temperature when the resistivity states are under $O$ and $F$. The inset illustrates the ferroelastic-domain states for the remnant strain states of $O$ and $F$, where ferroelectric polarization points to the out-of-plane direction ( $\mathrm{r}^{-}$and $\mathrm{r}^{-}$) and stays in the in-plane direction $\left(\mathrm{r}^{+} / \mathrm{r} 4^{+} / \mathrm{r} 3^{-} / \mathrm{r} 4^{-}\right)$, respectively. field $E=+6.6 \mathrm{kV} / \mathrm{cm}$ is applied to the positively poled PIN-PMN-PT substrate and returns to its initial value upon the removal of the electric field. Such modulation is apparently due to the converse piezoelectric-effect-induced in-plane strain, as schematically illustrated in Fig. 6(d). When a positive $E=6.6 \mathrm{kV} / \mathrm{cm}$ is applied to the substrate, in-plane contraction will induce a reduction in the in-plane tensile strain and a larger out-of-plane lattice constant of the NNO film, as confirmed by the XRD results shown in Fig. 6(e) where the NNO (002) diffraction peak shifts toward a smaller $2 \theta$ angle under $6.6 \mathrm{kV} / \mathrm{cm}$.

\section{B. NNO/PMN-0.29PT(011)}

The electric-field modulation of the resistivity of NNO films with different voltage-induced ferroelastic-domainswitching pathways is measured at $T=300 \mathrm{~K}$ and is shown in Fig. 7(a). By applying a symmetrical bipolar electric field with an amplitude of $8 \mathrm{kV} / \mathrm{cm}$ to the PMN-PT(011) substrate, the resistivity of the NNO film displays a butterfly-like loop with respect to the electric field [see the green curve in Fig. 7(a)], which is similar to the shape of the strain vs electric field loop observed in the PMN-PT(011) substrate (see Fig. 8), indicating that the modulation of the resistivity of the NNO film can also be ascribed to the electric-field-induced strain effect. For symmetrical bipolar electric-field sweeping, the polarization that undergoes $109^{\circ}$ and $180^{\circ}$ switching at the coercive fields (see inset of Fig. 8) fails to create the distinct remnant strain due to the strain equivalence in these domain states. However, by cycling an asymmetrical bipolar electric field with a positive amplitude of $3.4 \mathrm{kV} / \mathrm{cm}$ (less than the coercive field) on the PMNPT(011) substrate, a hysteresis-like resistivity vs electric field curve is obtained, as shown by the pink curve in Fig. 7(a). Specifically, when an asymmetrical bipolar electric field is swept from 0 to $3.4 \mathrm{kV} / \mathrm{cm}$ on the negatively poled PMN-PT(011) substrate, the polarization undergoes $71^{\circ} / 109^{\circ}$ domain switching with the vectors changing from the two downward directions [corresponding to the $O$ strain state in the lower inset of Fig. 7(b)] to the four in-plane directions [corresponding to the $F$ strain state in the upper inset of Fig. 7(b)], leading to a significant in-plane tensile strain that can remain stable if the electric field returns from $3.4 \mathrm{kV} / \mathrm{cm}$ back to zero. Afterward, upon sweeping the electric field from 0 to $-8 \mathrm{kV} / \mathrm{cm}$, the in-plane polarization rotates back to the downward direction. Accordingly, the strain state recovers to its initial state once the electric field is removed [28,54,55]. Therefore, the domain-engineered FE/ferroelastic switching can be used for nonvolatile tuning of the resistivity in NNO/PMN-PT(011) structures.

In addition to the electric-field-induced modulation of the resistivity of the NNO film near room temperature, the metal-to-insulator transition temperature $\left(T_{\mathrm{MI}}\right)$ of the NNO film can also be modified by domain-engineered

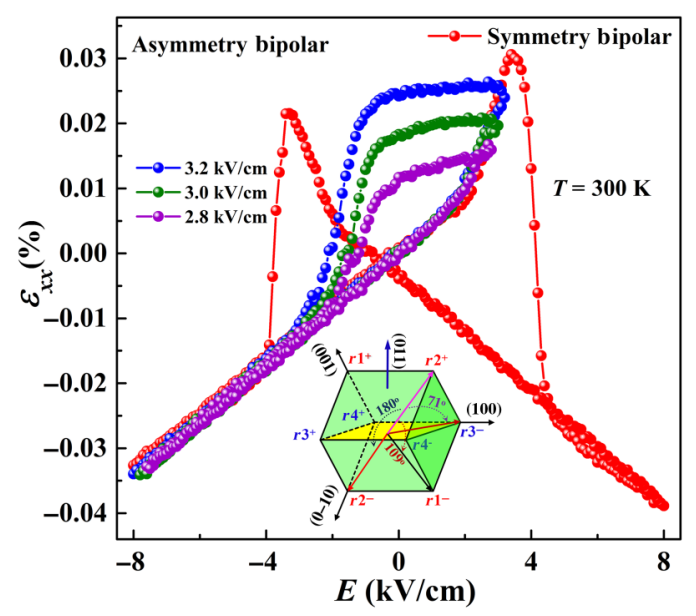

FIG. 8. In-plane strain of PMN-PT(011) substrate at $T=300 \mathrm{~K}$ as a function of symmetrical electric field and different amplitudes of asymmetrical electric field. The inset is the schematics of $109^{\circ}, 71^{\circ}$, and $180^{\circ}$ polarization switching induced by applying a negative voltage to a positively poled PMN-PT(011) substrate. 
(a)

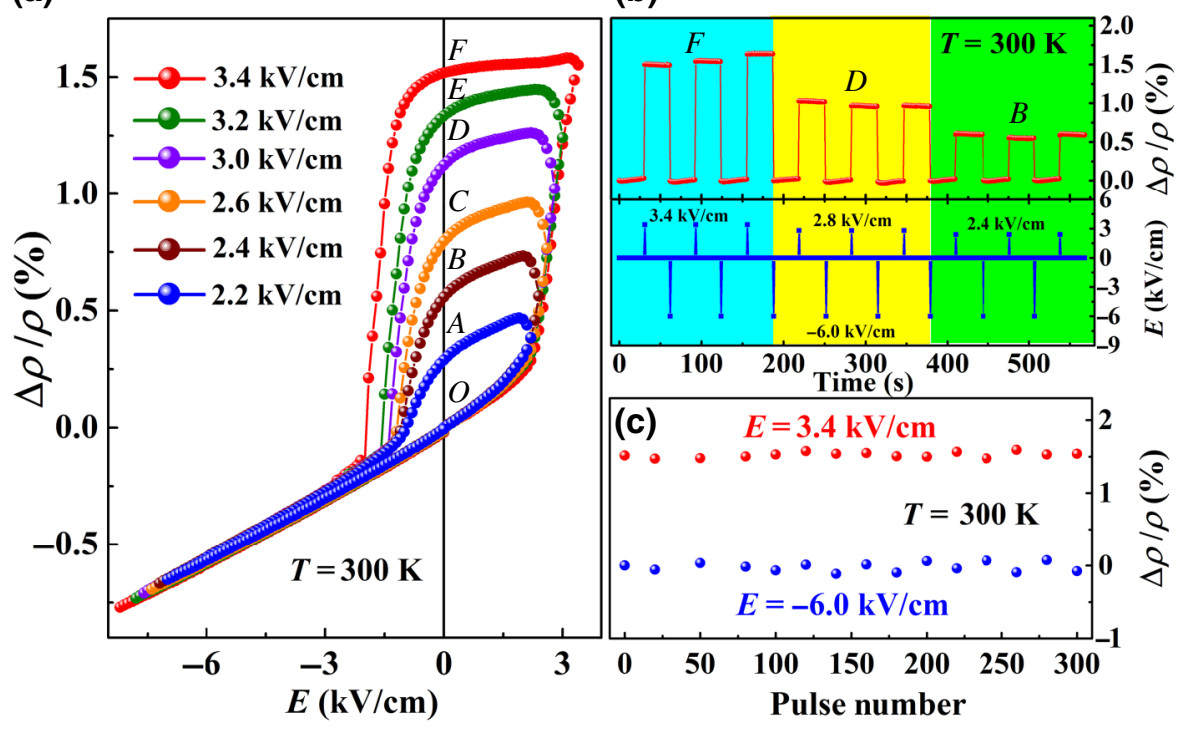

FIG. 9. (a) Relative resistivity change $(\Delta \rho / \rho)$ as a function of asymmetrical electric field with different amplitudes applied to the PMN-PT(011) substrate. (b) The nonvolatile resistance switching of the NNO film by a sequence of pulse electric fields at $T=300 \mathrm{~K}$. (c) The endurance characteristics of the two resistance states as a function of switching cycles for the NNO/PMN-PT(011) structure.
FE/ferroelastic switching through the application of an electric field with an appropriate magnitude. As shown in Fig. 7(b), both of the resistivity vs temperature curves display hysteresis between cooling and heating cycles. Moreover, the $T_{\mathrm{MI}}$ of the NNO film can be notably increased by approximately $15 \mathrm{~K}$. By switching the strain state of PMN$\mathrm{PT}(011)$ substrate from $O$ state to $F$ state by an electric field, an in-tensile strain is generated due to the $71^{\circ} / 109^{\circ}$ ferroelastic domain switching $[28,55]$, which is simultaneously transferred to the NNO film across the interface. Earlier, it was reported that a large in-plane compressive strain closes the charge-transfer gap and melts the insulating state of NNO film [14], leading to the decrease of $T_{\mathrm{MI}}$. In our study, the stain state transformation from $O$ to $F$ leads to in-plane tensile strain, thereby resulting in the increase of $T_{\mathrm{MI}}$.

Figure 9(a) shows the hysteretic responses of the resistivity of the NNO film at $T=300 \mathrm{~K}$ by cycling different amplitudes of asymmetrical bipolar electric fields on the PMN-PT(011) substrate. It is obvious that seven resistivity states corresponding to $O, A, B, C, D, E, F$ are obtained by modulating the amplitude of electric fields with $0,2.2$, $2.4,2.6,2.8,3.0$, and $3.4 \mathrm{kV} / \mathrm{cm}$, respectively. The maximum relative resistivity change of approximately $1.52 \%$ is obtained near the $E_{\mathrm{C}}$ of the PMN-PT(011) substrate $(-3.4 \mathrm{kV} / \mathrm{cm})$. On the basis of these results, multiple resistivity states of the NNO film can be reversibly switched through the application of appropriate electric-field pulses to the NNO/PMN-PT(011) heterostructure, as shown in Fig. 9(b). First, an electric-field pulse of $3.4 \mathrm{kV} / \mathrm{cm}$ is applied to the heterostructure and the resistivity state of the NNO film is switched from $O$ to $F$. Further, through modulating the amplitude of positive-electric-field pulses to $2.6 \mathrm{kV} / \mathrm{cm}$ or $2.4 \mathrm{kV} / \mathrm{cm}$, the resistivity states $D$ and $B$ of the NNO film can be achieved.
From a practical application point of view, the reliability and stability of resistivity states are important for a memory device to maintain its functionality. Thus, we perform pulse endurance measurements. A cycled electric field pulse sequence of -6 and $3.4 \mathrm{kV} / \mathrm{cm}$ is alternately applied to the PMN-PT(011) substrate, as shown in Fig. 9(c). It is noted that the values of these two resistivity states are quite stable and can be maintained up to 300 cycles without noticeable degradation. Therefore, the resistivity states in this heterostructure are nonvolatile, which may have potential applications in designing memory devices [28,55-58].

\section{NNO/PMN-PT(111)}

Figure 10(a) shows the relative resistivity change $(\Delta \rho / \rho)$ of the NNO film and the in-plane stain of the PMN-PT(111) substrate as a function of the electric field applied along the thickness direction of the PMN-PT(111) substrate [see inset of Fig. 10(a) for schematic illustration]. Note that the PMN-PT(111) substrate is in the unpoled virgin state before applying electric fields. Here, $\Delta \rho / \rho$ is defined as $\Delta \rho / \rho=[\rho(E)-\rho(0)] / \rho(0)$ where $\rho(E)$ and $\rho(0)$ are the resistivities of the NNO film when an electric field and zero electric field are applied to the PMN-PT(111) substrate. $\Delta \rho / \rho$ is nearly field independent for $E<2.8 \mathrm{kV} / \mathrm{cm}$ and decreases dramatically in the electric-field range of $2.8 \mathrm{kV} / \mathrm{cm}<E<3.5 \mathrm{kV} / \mathrm{cm}$. For $E>3.5 \mathrm{kV} / \mathrm{cm}$, the resistivity remains almost constant. These resistivity changes follow the electric-fieldinduced in-plane compressive strain of the PMN-PT(111) substrate [see the red curve in Fig. 10(a)] quite well, indicating that the electronic transport of the NNO film is closely related to its strain state. It is known that the rhombohedral PMN-PT(111) single crystals have eight 
(a)

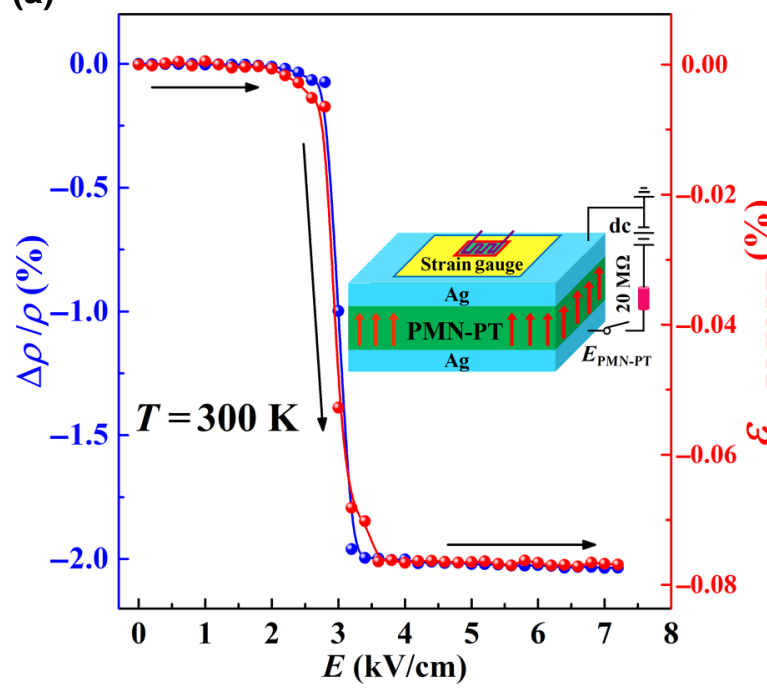

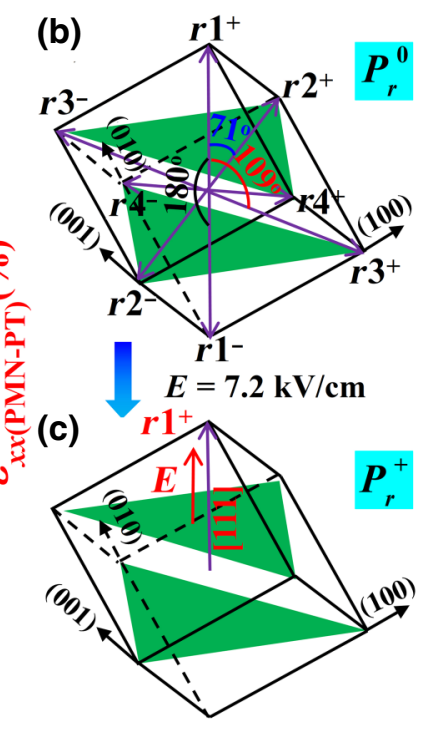

FIG. 10. (a) Relative resistivity changes $(\Delta \rho / \rho)$ of the NNO film (blue) and the electric-field-induced in-plane strain of the PMN-PT(111) substrate (red) as a function of electric field applied to the PMN-PT(111) substrate. Inset: Schematic of the experimental setup for the measurements of the electric-field-induced in-plane strain in the PMN-PT(111) substrate. The red arrows represent the polarization direction. (b),(c) The schematic diagrams of the $71^{\circ}, 109^{\circ}$, and $180^{\circ}$ polarization switching for the $P_{r}{ }^{0}(E=0 \mathrm{kV} / \mathrm{cm})$ and $P_{r}{ }^{+}(E=7.2 \mathrm{kV} / \mathrm{cm})$ states for the PMN-PT(111). spontaneous FE polarization directions along the body diagonals in the unpoled $P_{r}^{0}$ state [Fig. 10(b)]. With increasing $E$ from 0 to $7.2 \mathrm{kV} / \mathrm{cm}$, the $71^{\circ}$ ferroelastic domain (from $\mathrm{r} 2^{+} / \mathrm{r} 3^{-} / \mathrm{r}^{+}$to $\mathrm{r}^{+}$), $109^{\circ}$ ferroelastic domain (from r2 $2^{-} / \mathrm{r}^{+} / \mathrm{r}^{-}$to $\mathrm{r}^{+}$), and $180^{\circ}$ ferroelastic domain (from $\mathrm{rl}^{-}$to $\mathrm{rl}^{+}$) rotate to an upward direction and point along the [111] crystal direction [Fig. 10(c)] without an in-plane component since the PMN-PT(111) single crystal is under 3-m symmetry [59].

Further, we measure hysteretic responses of the resistivity of NNO film at $T=300 \mathrm{~K}$ by applying a symmetrical bipolar electric field with an amplitude of $8 \mathrm{kV} / \mathrm{cm}$ and cycling different amplitudes of asymmetrical bipolar electric fields on the PMN-PT(111) substrate and show the (a)

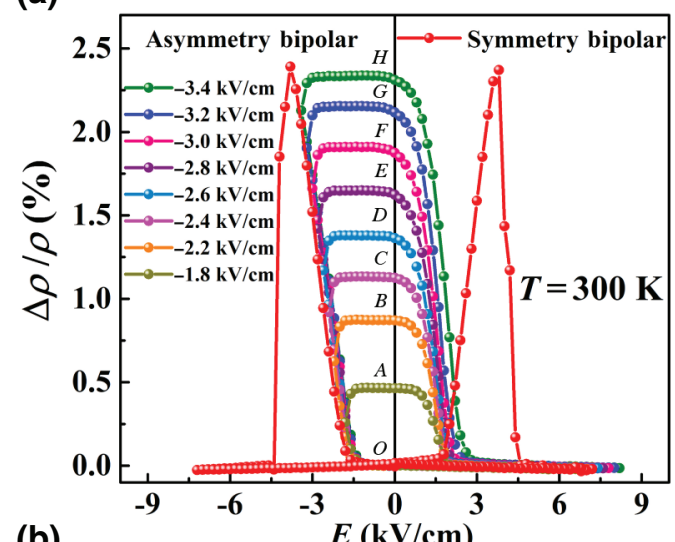

(b)

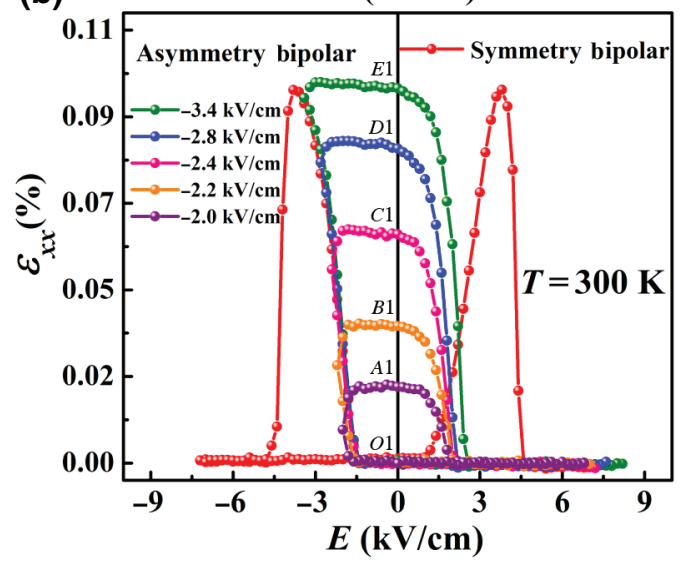

(c)

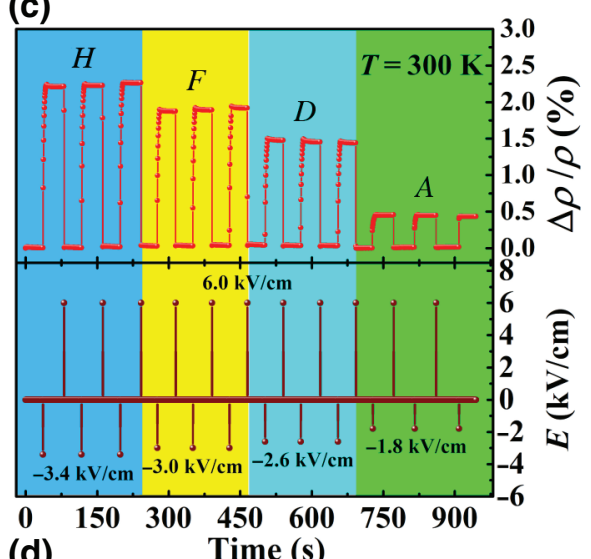

(d)

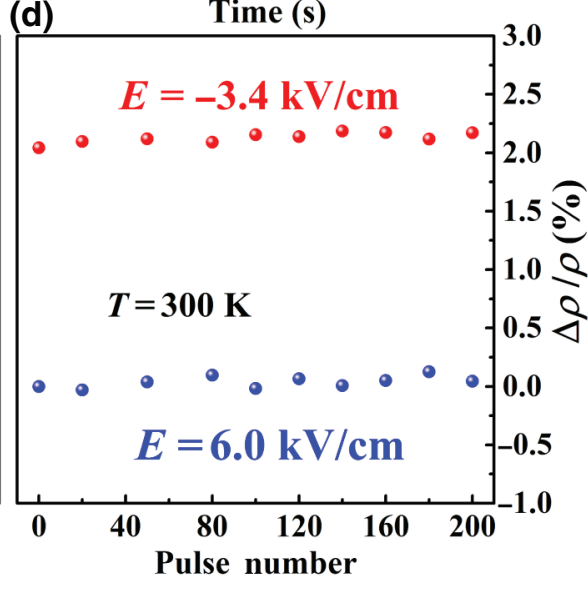

FIG. 11. (a) Relative resistivity change $(\Delta \rho / \rho)$ of the NNO film at $T=300 \mathrm{~K}$ as a function of symmetrical and asymmetrical electric fields applied to the PMN-PT(111) substrate. (b) Inplane strain of PMN-PT(111) substrate at $T=300 \mathrm{~K}$ as a function of symmetrical and asymmetrical electric fields. (c) Nonvolatile resistance switching of the NNO film by a sequence of pulsed electric fields at $T=300 \mathrm{~K}$. (d) The endurance characteristics of the two resistance states as a function of switching cycles for the NNO/PMN-PT(111) structure. 
(a)

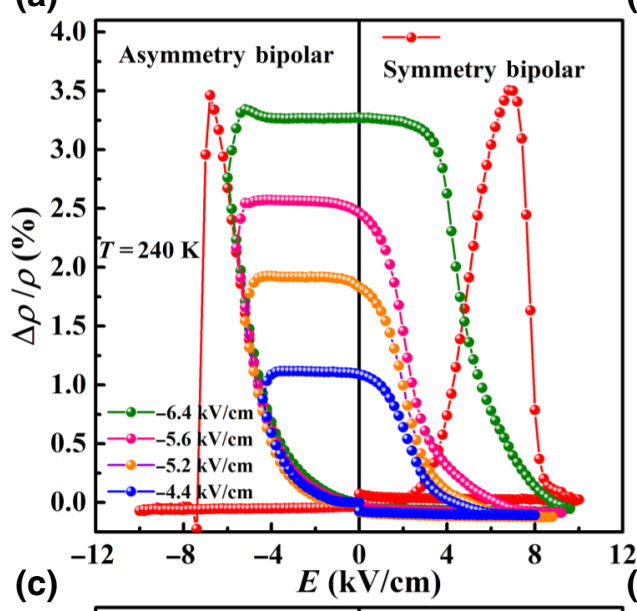

(b)

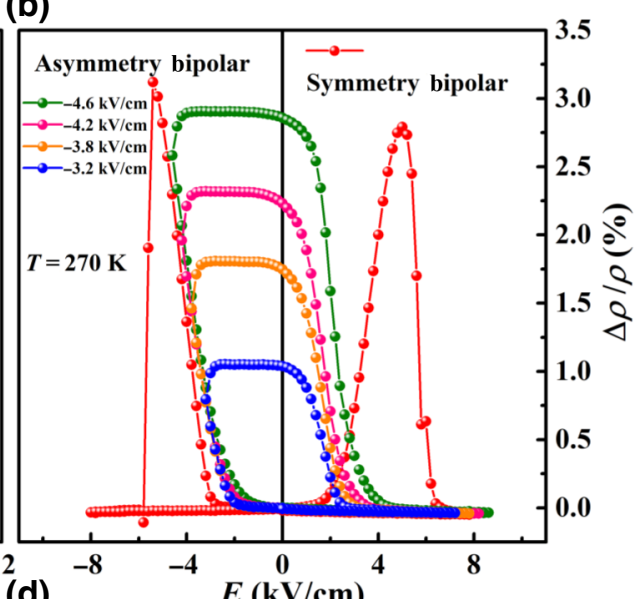

(d)

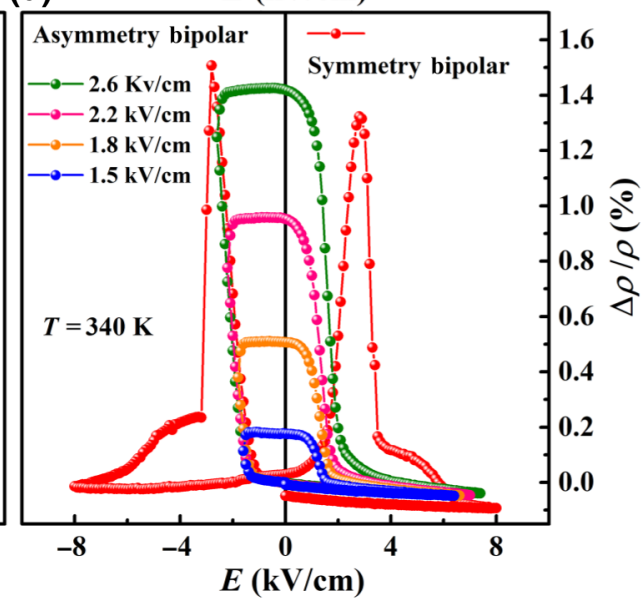

(kV/cm)
FIG. 12. Relative resistivity changes $(\Delta \rho / \rho)$ of the NNO film at various fixed temperatures as a function of a symmetrical electric field and different amplitudes of asymmetrical electric field applied to the PMN-PT(111) substrate.

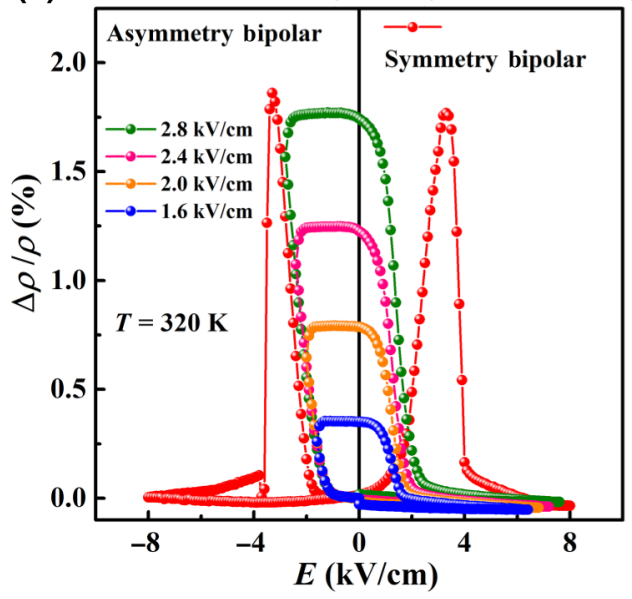

results in Fig. 11(a). The resistivity of the NNO film displays a butterfly-like loop with the symmetrical bipolar electric field, which is quite similar to the shape of strain $\left(\varepsilon_{x x}\right)$ vs electric-field loop [Fig. 11(b)], further indicating that the modulation of the resistivity in the NNO film is due to the electric-field-induced strain. For the symmetrical bipolar electric field, the remnant resistivity state cannot be obtained due to the $180^{\circ} \mathrm{FE}$-domain switching, which results in zero remnant strain. However, multiple resistivity states can be obtained by adjusting the amplitude of asymmetric electric fields [Fig. 11(a)]. The maximum relative resistivity change of approximately $2.34 \%$ is obtained near the $E_{\mathrm{C}}$ of the PMN-PT(111) substrate $(-3.4 \mathrm{kV} / \mathrm{cm})$. By applying $-3.4 \mathrm{kV} / \mathrm{cm}$ and $8 \mathrm{kV} / \mathrm{cm}$ electric fields, two remnant resistivity states corresponding to $O$ and $H$ are obtained [Fig. 11(a)]. It is obvious that the resistivity does not return to its initial state when the electric field returns back to zero unless a positive electric field is applied, showing a nonvolatile switching feature. Further, the other seven resistivity states corresponding to $A, B, C, D, E, F$, and $G$ are obtained by modulating the amplitude of the electric fields with $-1.8,-2.0,-2.2,-2.4,-2.6,-2.8$, -3.0 , and $-3.2 \mathrm{kV} / \mathrm{cm}$, respectively [Fig. 11(a)]. It is noted that multiple resistivity states can also achieved with several other temperatures for the NNO/PMN-PT(111) heterostructure (see Fig. 12). In fact, more resistivity states can be achieved by precisely controlling the magnitude of the electric fields. To further understand the observed nonvolatile resistivity modulation more clearly, the in-plane strain $\varepsilon_{x x}$ of the PMN-PT(111) substrate with different amplitudes of asymmetric electric fields at $T=300 \mathrm{~K}$ is measured, as shown in Fig. 11(b). By applying 8.0, -2.0, $-2.2,-2.4,-2.8$, and $-3.4-\mathrm{kV} / \mathrm{cm}$ electric fields to the PMN-PT(111) substrate, six strain states corresponding to $O 1, A 1, B 1, C 1, D 1$, and $E 1$ can be obtained. The multiple strain states can also be achieved with other several temperatures for the PMN-PT(111) substrate (see Fig. 13). On the basis of these results, multiple resistivity states of the NNO film can also be reversibly switched through the application of appropriate electric field pulses to the NNO/PMN-PT(111) heterostructure, as shown in Fig. 11(c). First, an electric-field pulse of $-3.4 \mathrm{kV} / \mathrm{cm}$ is applied to the heterostructure and the resistivity state of the NNO film is switched from $O$ to $H$. Further, through modulating the amplitude of negative-electric-field pulses to $-3.0,-2.6$, or $-1.8 \mathrm{kV} / \mathrm{cm}$ without varying the positiveelectric-field pulses, the resistivity states $F, D$, and $A$ are achieved. 
(a)

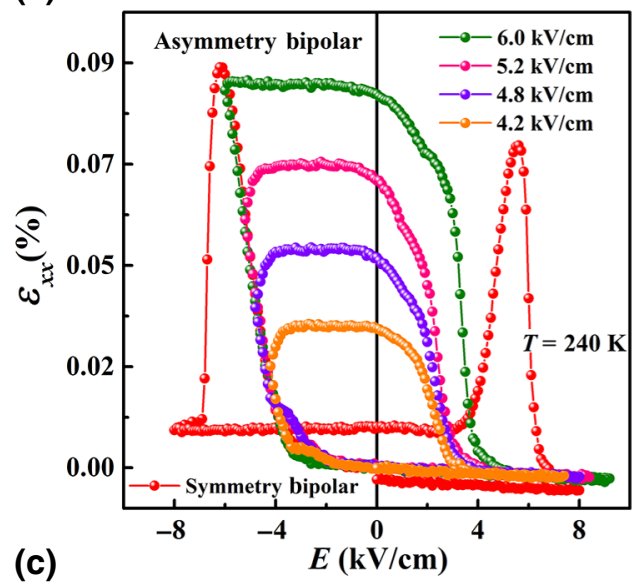

(c)

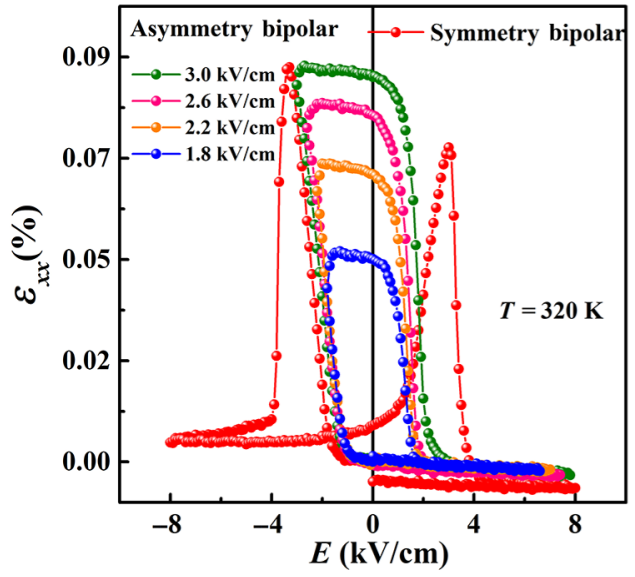

(b)

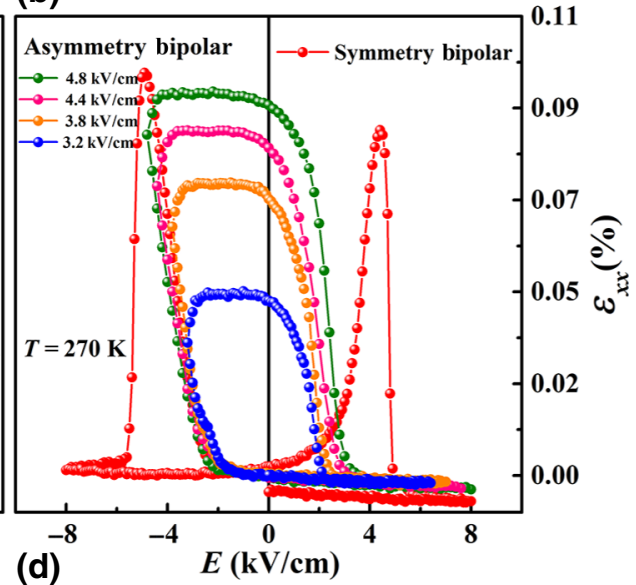

(d)

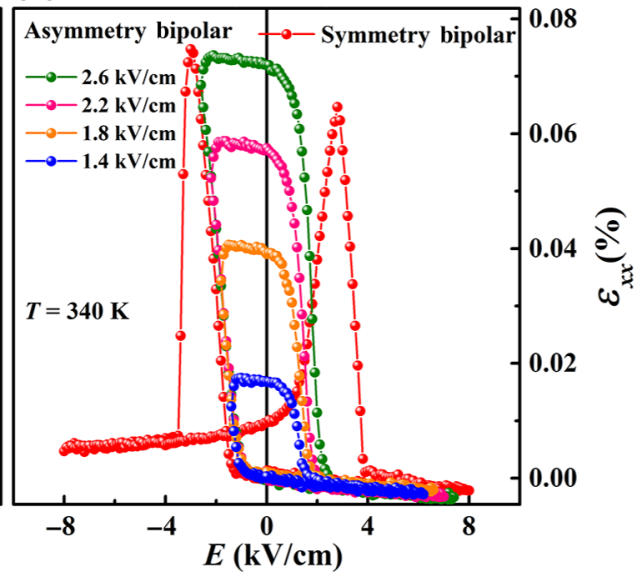

FIG. 13. In-plane strain of the PMN-PT(111) substrate as a function of symmetrical electric field and different amplitudes of asymmetrical electric field at several fixed temperatures.
Again, we perform pulse endurance measurements on the NNO/PMN-PT(111) heterostructure. A cycled electricfield-pulse sequence of +6 and $-3.4 \mathrm{kV} / \mathrm{cm}$ is alternately applied to the PMN-PT(111) substrate, as shown in Fig. 11(d). The two resistivity states are quite stable and can be maintained for at least 200 cycles without noticeable degradation. Therefore, the resistivity states in this heterostructure are also nonvolatile, which may have potential applications in designing low-power-consuming memory devices [55-57].

Taking advantage of the remnant resistivity state of the NNO film grown on the PMN-PT(111) substrate [inset (I) of Fig. 14(a)], we measure the resistivity of the NNO film under $P_{r}^{O}$ (red curve) and $P_{r}^{H}$ (blue curve) states [Fig.

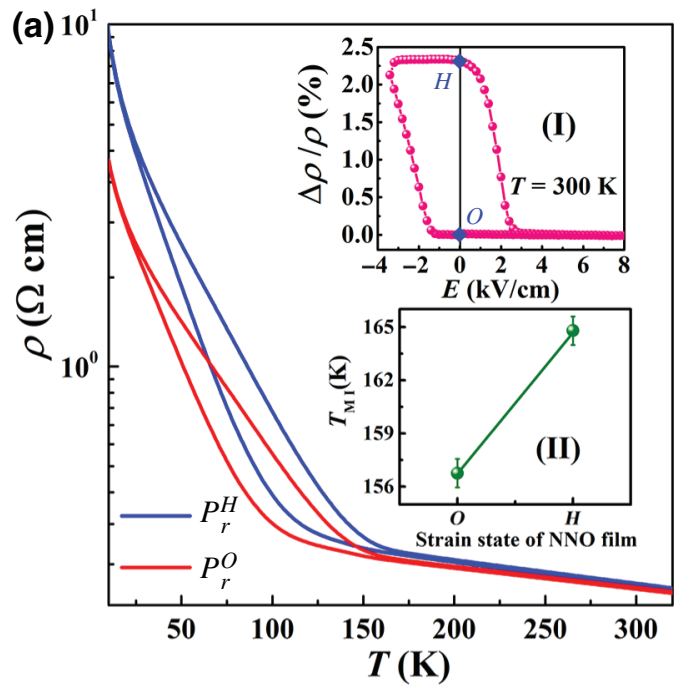

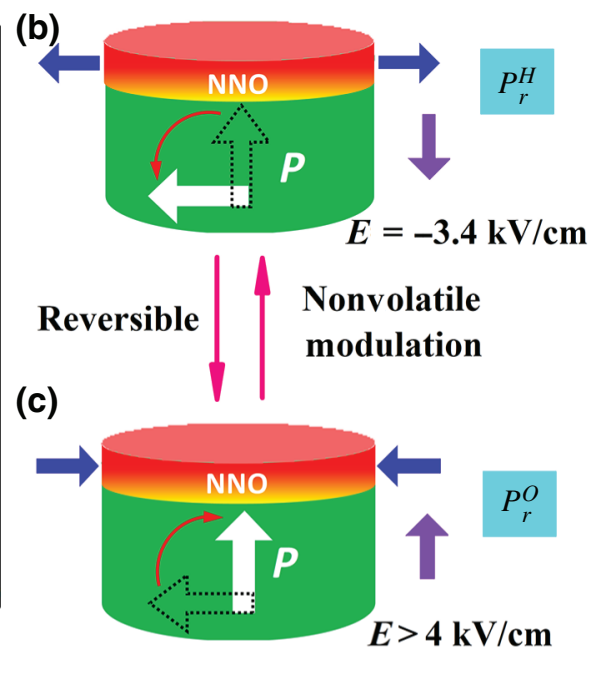

FIG. 14. (a) The variation of resistivity with temperature under $P_{r}^{O}$ and $P_{r}^{H}$ states. Inset (I): The relative resistivity change $(\Delta \rho / \rho)$ of the NNO film at $T=300 \mathrm{~K}$ as a function of the asymmetrical electric field applied to the PMNPT(111) substrate. Inset (II): $T_{\mathrm{MI}}$ of the NNO film under $P_{r}^{O}$ and $P_{r}^{H}$ states. (b),(c) Schematic diagrams for the polarizationrotation-induced in-plane strain in the NNO film. 
14(a)]. $T_{\mathrm{MI}}$ of the NNO film can be modified by switching the resistivity state from $O$ to $H$. As discussed in previous sections, the switching of the resistivity state from $O$ to $H$ is, in fact, due to the ferroelastic-domain rotation-induced in-plane tensile strain in the NNO film [see Figs. 14(b) and 14(c) for schematic illustration]. Such an induced in-plane tensile strain results in an increase in $T_{\mathrm{MI}}$ (approximately $5.1 \%$ ) [see inset (II) of Fig. 14(a)], which is consistent with the results that were discussed in the previous sections concerning the substrate-induced static strain effect and electric-field-induced strain effects in a NNO/PMNPT(011) structure. The stable and reversible in-plane and out-of-plane polarization states of the PMN-PT(111) substrate enable the control of the transition temperature of the NNO film by simply switching the proper electric-field pulse, which offers an energy efficient way to control the metal-to-insulator transition of NNO films.

\section{CONCLUSIONS}

In summary, $\mathrm{NNO}$ films are epitaxially grown on a set of substrates, including the piezoelectrically active PIN-PMN-PT and PMN-PT single-crystal substrates. On moving from tensile to compressive strain, electronic transport measurements demonstrate a successively suppressed metal-to-insulator transition, which eventually disappears at a high compressive strain. Corroborating these findings, we in situ impose in-plane compressive/tensile strain to NNO films via the electric-field-induced ferroelastic strain of PIN-PMN-PT and PMN-PT substrates and achieve a large resistivity modulation up to $125 \%$ near the metal-to-insulator transition temperature. Using the electric-field-tunable dynamic piezostrain, we establish a quantitative relationship between the resistivity and out-of-plane strain of the NNO films, with a gauge factor $(\Delta \rho / \rho) / \delta \varepsilon_{x x} \sim 40.8$. Taking advantage of the domain-engineered FE/ferroelastic switching of the PMNPT substrate, multistate stable resistivity states with good retention and endurance properties are obtained at room temperature and the metal-to-insulator transition temperature $\left(T_{\mathrm{MI}}\right)$ of the NNO film can be modified by precisely controlling the magnitude of the electric-field-pulse sequence. Our results demonstrate that the electric-fieldtunable ferroelastic (particularly at low temperatures) and piezoelectric strain approach can be utilized to gain deeper insight into the intrinsic and quantitative relationship between strain and electronic properties of nickelates and provide a simple and energy-efficient way to construct multistate resistive memory.

\section{ACKNOWLEDGMENTS}

This work was supported by the National Natural Science Foundation of China (Grants No. 51572278, No. 51790491, and No. 51872278), the National Basic Research Program of China (Grants No. 2016YFA0300103 and No. 2015CB921201) and the opening project of State Key Laboratory of High Performance Ceramics and Superfine Microstructure, Shanghai Institute of Ceramics, Chinese Academy of Sciences (Grant No. SKL201708SIC). Support from Jiangxi Key Laboratory for Two-Dimensional Materials and Jiangxi Engineering Laboratory for Thin Films and Devices is also acknowledged. M.M. and R.K. thank Professor X.M. Li for his partial support in the preparation of the NNO films.

J.-M. Y., M. X. and T.-W. C. contributed equally to this work.

[1] P. A. Lee, N. Nagaosa, and X. G. Wen, Doping a Mott insulator: Physics of high-temperature superconductivity, Rev. Mod. Phys. 78, 17 (2006).

[2] W. Eerenstein, N. D. Mathur, and J. F. Scott, Multiferroic and magnetoelectric materials, Nature 442, 759 (2006).

[3] M. Imada, A. Fujimori, and Y. Tokura, Metal-to-insulator transitions, Rev. Mod. Phys. 70, 1039 (1998).

[4] J. B. Torrance, P. Lacorre, A. I. Nazzal, E. J. Ansaldo, and C. Niedermayer, Systematic study of insulator-metal transitions in perovskites $R \mathrm{NiO}_{3}(\mathrm{R}=\mathrm{Pr}, \mathrm{Nd}, \mathrm{Sm}, \mathrm{Eu})$ due to closing of charge-transfer gap, Phys. Rev. B 45, 8209 (1992).

[5] G. Catalan, Progress in perovskite nickelate research, Phase Trans. 81, 729 (2008).

[6] I. Kézsmárki, Y. Tomioka, S. Miyasaka, L. Demkó, Y. Okimoto, and Y. Tokura, Optical phase diagram of perovskite colossal magnetoresistance manganites near half doping, Phys. Rev. B 77, 075117 (2008).

[7] J. L. García-Muñoz, M. Suaaidi, M. J. Martínez-Lope, and J. A. Alonso, Influence of carrier injection on the metal-toinsulator transition in electron-and hole-doped $R_{1-\mathrm{x}} A_{\mathrm{x}} \mathrm{NiO}_{3}$ perovskites, Phys. Rev. B 52, 13563 (1995).

[8] X. Obradors, L. M. Paulius, M. B. Maple, J. B. Torrance, A. I. Nazzal, J. Fontcuberta, and X. Granados, Pressure dependence of the metal-to-insulator transition in the chargetransfer oxides $\mathrm{RNiO}_{3}\left(R=\mathrm{Pr}, \mathrm{Nd}, \mathrm{Nd}_{0.7} \mathrm{La}_{0.3}\right)$, Phys. Rev. B 47, 12353 (1993).

[9] P. C. Canfield, J. D. Thompson, S.-W. Cheong, and L. W. Rupp, Extraordinary pressure dependence of the metalto-insulator transition in the charge-transfer compounds $\mathrm{NdNiO}_{3}$ and $\mathrm{PrNiO}_{3}$, Phys. Rev. B 47, 12357 (1993).

[10] R. Scherwitzl, P. Zubko, I. G. Lezama, S. Ono, A. F. Morpurgo, G. Catalan, and J. M. Triscone, Electric-field control of the metal-to-insulator transition in ultrathin $\mathrm{NdNiO}_{3}$ films, Adv. Mater. 22, 5517 (2010).

[11] G. Catalan, R. M. Bowman, and J. M. Gregg, Metal-toinsulator transitions in $\mathrm{NdNiO}_{3}$ thin films, Phys. Rev. B 62 , 7892 (2000).

[12] Y. Kumar, R. J. Choudhary, and R. Kumar, Strain controlled systematic variation of metal-to-insulator transition in epitaxial $\mathrm{NdNiO}_{3}$ thin films, J. Appl. Phys. 112, 073718 (2012).

[13] Y. Kumar, R. J. Choudhary, S. K. Sharma, M. Knobel, and R. Kumar, Strain dependent stabilization of metallic paramagnetic state in epitaxial $\mathrm{NdNiO}_{3}$ thin films, Appl. Phys. Lett. 101, 132101 (2012). 
[14] J. Liu, M. Kareev, B. Gray, J. W. Kim, P. Ryan, B. Dabrowski, J. W. Freeland, and J. Chakhalian, Strainmediated metal-to-insulator transition in epitaxial ultrathin films of $\mathrm{NdNiO}_{3}$, Appl. Phys. Lett. 96, 233110 (2010).

[15] X. K. Lian, F. Chen, X. L. Tan, P. F. Chen, L. F. Wang, G. Y. Gao, S. W. Jin, and W. B. Wu, Anisotropic-straincontrolled metal-to-insulator transition in epitaxial $\mathrm{NdNiO}_{3}$ films grown on orthorhombic $\mathrm{NdGaO}_{3}$ substrates, Appl. Phys. Lett. 103, 172110 (2013).

[16] R. Eguchi, Y. Okamoto, Z. Hiroi, S. Shin, A. Chainani, Y. Tanaka, M. Matsunami, Y. Takata, Y. Nishino, K. Tamasaku, M. Yabashi, and T. Ishikawa, Structure and photoemission spectroscopy of strain-controlled metal-toinsulator transition in $\mathrm{NdNiO}_{3}$ thin films, J. Appl. Phys. 105, 056103 (2009).

[17] L. Shu, T. Li, Z. Wang, F. Li, L. Fei, Z. Rao, M. Ye, S. Ke, W. Huang, Y. Wang, and X. Yao, Flexoelectric behavior in PIN-PMN-PT single crystals over a wide temperature range, Appl. Phys. Lett. 111, 162901 (2017).

[18] G. Xu, K. Chen, D. Yang, and J. Li, Growth and electrical properties of large size $\mathrm{Pb}\left(\mathrm{In}_{1 / 2} \mathrm{Nb}_{1 / 2} \mathrm{O}_{3}-\right.$ $\mathrm{PbMg}_{1 / 3} \mathrm{Nb}_{2 / 3} \mathrm{O}_{3}-\mathrm{PbTiO}_{3}$ crystals prepared by the vertical Bridgman technique, Appl. Phys. Lett. 90, 032901 (2007).

[19] X. Li, Z. Wang, C. He, X. Long, and Z. G. Ye, Growth and piezo-/ferroelectric properties of PIN-PMN-PT single crystals, J. Appl. Phys. 111, 034105 (2012).

[20] Y. Hosono, Y. Yamashita, H. Sakamoto, and N. Ichinose, Large piezoelectric constant of high-curie-temperature $\mathrm{Pb}\left(\mathrm{In}_{1 / 2} \mathrm{Nb}_{1 / 2}\right) \mathrm{O}_{3}-\mathrm{Pb}\left(\mathrm{Mg}_{1 / 3} \mathrm{Nb}_{2 / 3}\right)-\mathrm{PbTiO}_{3}$ ternary single crystal near morphotropic phase boundary, Jpn. J. Appl. Phys. 41, L1240 (2002).

[21] M. Zheng, M. M. Yang, Q. X. Zhu, X. Y. Li, G. Y. Gao, R. K. Zheng, Y. Wang, X. M. Li, X. Shi, H. S. Luo, and $\mathrm{X}$. G. Li, Tunable interface strain coupling and its impact on the electronic transport and magnetic properties of $\mathrm{La}_{0.5} \mathrm{Ca}_{0.5} \mathrm{MnO}_{3} / \mathrm{Pb}\left(\mathrm{In}_{1 / 2} \mathrm{Nb}_{1 / 2}\right) \mathrm{O}_{3}-\mathrm{Pb}\left(\mathrm{Mg}_{1 / 3} \mathrm{Nb}_{2 / 3}\right) \mathrm{O}_{3}$ $\mathrm{PbTiO}_{3}$ multiferroic heterostructures, Phys. Rev. B 90, 224420 (2014).

[22] C. Thiele, K. Dörr, S. Fähler, L. Schultz, D. C. Meyer, A. A. Levin, and P. Paufler, Voltage-controlled epitaxial strain in $\mathrm{La}_{0.7} \mathrm{Sr}_{0.3} \mathrm{MnO}_{3} / \mathrm{PbMg}_{1 / 3} \mathrm{Nb}_{2 / 3} \mathrm{O}_{3}-\mathrm{PbTiO}_{3}$ (001) films, Appl. Phys. Lett. 87, 262502 (2005).

[23] R. K. Zheng, Y. Wang, H. L. W. Chan, C. L. Choy, and H. S. Luo, Substrate-induced strain effect in $\mathrm{La}_{0.875} \mathrm{Ba}_{0.125} \mathrm{MnO}_{3}$ thin films grown on ferroelectric single-crystal substrates, Appl. Phys. Lett. 92, 082908 (2008).

[24] M. Zheng and R. K. Zheng, Electric-Field-Tunable Ferroelastic Control of Nonvolatile Resistivity and Ferromagnetic Switching in Multiferroioc $\mathrm{La}_{0.67} \mathrm{Ca}_{0.33} \mathrm{MnO}_{3} /\left[\mathrm{PbMg}_{1 / 3}\right.$ $\left.\mathrm{Nb}_{2 / 3} \mathrm{O}_{3}\right]_{0.7}\left[\mathrm{PbTiO}_{3}\right]_{0.3}$ Heterostructures, Phys. Rev. Appl. 5, 044002 (2016).

[25] Y. Y. Zhao, J. Wang, F. X. Hu, H. Kuang, R. R. Wu, X. Q. Zheng, J. R. Sun, and B. G. Shen, Influence of lattice strain on charge/orbital ordering and phase separation in $\operatorname{Pr}_{0.7}\left(\mathrm{Ca}_{0.6} \mathrm{Sr}_{0.4}\right)_{0.3} \mathrm{MnO}_{3}$ thin films, J. Appl. Phys. 115, 17D708 (2014).

[26] R. K. Zheng, Y. Jiang, Y. Wang, H. L. W. Chan, C. L. Choy, and H. S. Luo, Ferroelectric poling and converse-piezoelectric-effect-induced strain effects in
$\mathrm{La}_{0.7} \mathrm{Ba}_{0.3} \mathrm{MnO}_{3}$ thin films grown on ferroelectric singlecrystal substrates, Phys. Rev. B 79, 174420 (2009).

[27] M. Zheng, H. Ni, X. Xu, Y. Qi, X. Li, and J. Gao, Optically Tunable Resistive-Switching Memory in Multiferroic Heterostructures, Phys. Rev. Appl. 9, 044039 (2018).

[28] W. Zhou, Y. Xiong, Z. Zhang, D. Wang, W. Tan, Q. Cao, Z. Qian, and Y. Du, Multilevel resistance switching memory in $\mathrm{La}_{2 / 3} \mathrm{Ba}_{1 / 3} \mathrm{MnO}_{3} / 0.7 \mathrm{~Pb}\left(\mathrm{Mg}_{1 / 3} \mathrm{Nb}_{2 / 3}\right) \mathrm{O}_{3}-0.3 \mathrm{PbTiO}_{3} \quad(011)$ heterostructure by combined straintronics-spintronics, ACS Appl. Mater. Interfaces 8, 5424 (2016).

[29] W. Zhao, D. Zhang, D. Meng, W. Huang, L. Feng, C. Hou, Y. Lu, Y. Yin, and X. G. Li, Electric-field-controlled nonvolatile magnetic switching and resistive change in $\mathrm{La}_{0.6} \mathrm{Sr}_{0.4} \mathrm{MnO}_{3} / 0.7 \mathrm{~Pb}\left(\mathrm{Mg}_{1 / 3} \mathrm{Nb}_{2 / 3}\right) \mathrm{O}_{3}-0.3 \mathrm{PbTiO}_{3} \quad$ (011) heterostructure at room temperature, Appl. Phys. Lett. 109, 263502 (2016).

[30] Q. Yang, T. Nan, Y. Zhang, Z. Zhou, and B. Peng, Voltage Control of Perpendicular Magnetic Anisotropy in Multiferroic $(\mathrm{Co}=\mathrm{Pt})_{3} / \mathrm{PbMg}_{1 / 3} \mathrm{Nb}_{2 / 3} \mathrm{O}_{3}-\mathrm{PbTiO}_{3}$ Heterostructures, Phys. Rev. Appl. 8, 044006 (2017).

[31] C. Zhou, L. Shen, M. Liu, C. Gao, C. Jia, and C. Jiang, Strong Nonvolatile Magnon-Driven Magnetoelectric Coupling in Single-Crystal $\mathrm{Co} /\left[\mathrm{PbMg}_{1 / 3} \mathrm{Nb}_{2 / 3} \mathrm{O}_{3}\right]_{0.71}\left[\mathrm{PbTiO}_{3}\right]_{0.29}$ Heterostructures, Phys. Rev. Appl. 9, 014006 (2018).

[32] W. Zhang, M. M. Yang, X. Liang, H. W. Zheng, Y. Wang, W. X. Gao, G. L. Yuan, W. F. Zhang, X. G. Li, H. S. Luo, and R. K. Zheng, Piezostrain-enhanced photovoltaic effects in $\mathrm{BiFeO}_{3} / \mathrm{La}_{0.7} \mathrm{Sr}_{0.3} \mathrm{MnO}_{3} / \mathrm{PMN}-\mathrm{PT}$ heterostructures, Nano Energy 18, 315 (2015).

[33] R. Hühne, D. Okai, K. Dörr, S. Trommler, A. Herklotz, B. Holzapfel, and L. Schultz, Dynamic investigations on the influence of epitaxial strain on the superconducting transition in $\mathrm{YBa}_{2} \mathrm{Cu}_{3} \mathrm{O}_{7-x}$, Supercond. Sci. Technol. 21, 075020 (2008).

[34] Y. Yu, X. Zhang, J. W. W. and Y, and G. Zhao, Microstructure of epitaxial $\mathrm{YBa}_{2} \mathrm{Cu}_{3} \mathrm{O}_{7-\delta}$ thin films grown on $\mathrm{Pb}\left(\mathrm{Mg}_{1 / 3} \mathrm{Nb}_{2 / 3}\right)_{0.7} \mathrm{Ti}_{0.3} \mathrm{O}_{3}$ substrates, J. Cryst. Growth 354, 98 (2012).

[35] Z. Wu, Y. Zhang, G. Bai, W. Tang, J. Gao, and J. Hao, Effect of biaxial strain induced by piezoelectric PMN-PT on the upconversion photoluminescence of $\mathrm{BaTiO}_{3}: \mathrm{Yb} / \mathrm{Er}$ thin films, Opt. Express 22, 29014 (2014).

[36] F. Wang, D. Liu, Z. Chen, Z. Duan, Y. Zhang, D. Sun, X. Zhao, W. Shi, R. Zheng, and H. Luo, In situ reversible tuning of photoluminescence of an epitaxial thin film via piezoelectric strain induced by a $\mathrm{Pb}\left(\mathrm{Mg}_{1 / 3} \mathrm{Nb}_{2 / 3}\right) \mathrm{O}_{3}-\mathrm{PbTiO}_{3}$ single crystal, J. Mater. Chem. C 5, 9115 (2017).

[37] J. H. Park, Y. K. Jeong, S. Ryu, J. Y. Son, and H. M. Jang, Electric-field-control of magnetic remanence of $\mathrm{NiFe}_{2} \mathrm{O}_{4}$ thin film epitaxially grown on $\mathrm{PbMg}_{1 / 3} \mathrm{Nb}_{2 / 3} \mathrm{O}_{3}-\mathrm{PbTiO}_{3}$, Appl. Phys. Lett. 96, 192504 (2010).

[38] J. Y. Kim, L. Yao, and S. V. Dijken, Coherent piezoelectric strain transfer to thick epitaxial ferromagnetic films with large lattice mismatch, J. Phys.: Condens. Matter 25, 082205 (2013).

[39] A. Tkach, M. B. Yazdi, M. Foerster, F. Büttner, M. Vafaee, M. Fries, and M. Kläui, Magnetoelectric properties of epitaxial $\mathrm{Fe}_{3} \mathrm{O}_{4}$ thin films on (011) PMN-PT piezosubstrates, Phys. Rev. B 91, 024405 (2015). 
[40] Y. Zhang, M. Liu, L. Zhang, Z. Zhou, B. Peng, C. Wang, Q. Lin, Z. D. Jiang, W. Ren, and Z. G. Ye, Multiferroic heterostructures of $\mathrm{Fe}_{3} \mathrm{O}_{4} / \mathrm{PMN}-\mathrm{PT}$ prepared by atomic layer deposition for enhanced interfacial magnetoelectric couplings, Appl. Phys. Lett. 110, 082902 (2017).

[41] X. W. Zhao, G. Y. Gao, J. M. Yan, L. Chen, M. Xu, W. Y. Zhao, Z. X. Xu, L. Guo, Y. K. Liu, Y. Wang, and R. K. Zheng, Reversible and nonvolatile ferroelectric control of two-dimensional electronic transport properties of $\mathrm{ZrCuSiAs}$-type copper oxyselenide thin films with a layered structure, Phys. Rev. Mater 2, 055003 (2018).

[42] S. Heo, C. Oh, M. J. Eom, J. S. Kim, J. Ryu, J. Son, and H. M. Jang, Modulation of metal-to-insulator transitions by field-controlled strain in $\mathrm{NdNiO}_{3} / \mathrm{SrTiO}_{3} / \mathrm{PMN}-\mathrm{PT}(001)$ heterostructures, Sci. Rep. 6, 22228 (2016).

[43] See supplementary material at http://link.aps.org/supple mental/10.1103/PhysRevApplied.11.034037 for schematic of the experimental setup for measurements of resistance and electric-field-induced out-of-plane and in-plane strain in NNO film and PIN-PMN-PT and PMN-PT substrates, AFM image of the $25-\mathrm{nm} \mathrm{NdNiO}_{3}$ film on PIN-PMN-PT, temperature dependence of the dielectric constants for a positively poled PIN-PMN-PT substrate.

[44] R. K. Zheng, Y. Wang, H. L. W. Chan, C. L. Choy, and H. S. Luo, Determination of the strain dependence of resistance in $\mathrm{La}_{0.7} \mathrm{Sr}_{0.3} \mathrm{MnO}_{3} / \mathrm{PMN}-\mathrm{PT}$ using the converse piezoelectric effect, Phys. Rev. B 75, 212102 (2007).

[45] K. S. Takahashi, D. Matthey, D. Jaccard, J.-M. Triscone, K. Shibuya, T. Ohnishi, and M. Lippmaa, Electrostatic modulation of the electronic properties of $\mathrm{Nb}$-doped $\mathrm{SrTiO}_{3}$ superconducting films, Appl. Phys. Lett. 84, 1722 (2004).

[46] W. L. Lim, E. J. Moon, J. W. Freeland, D. J. Meyers, M. Kareev, J. Chakhalian, and S. Urazhdin, Field-effect diode based on electron-induced Mott transition in $\mathrm{NdNiO}_{3}$, Appl. Phys. Lett. 101, 143111 (2012).

[47] A. A. Bokov and Z.-G. Ye, Recent progress in relaxor ferroelectrics with perovskite structure, J. Mater. Sci. 41, 31 (2006).

[48] Y. Y. Zhao, J. Wang, H. Kuang, F. X. Hu, H. R. Zhang, Y. Liu, Y. Zhang, S. H. Wang, R. R. Wu, M. Zhang, L. F. Bao, J. R. Sun, and B. G. Shen, Abnormal percolative transport and colosal electroresistance induced by anisotropic strain in (011)- $\mathrm{Pr}_{0.7}\left(\mathrm{Ca}_{0.6} \mathrm{Sr}_{0.4}\right)_{0.3} \mathrm{MnO}_{3} / \mathrm{PMN}-$ PT heterostructure, Sci. Rep. 4, 7075 (2014).

[49] C. Adamo, X. Ke, H. Q. Wang, H. L. Xin, T. Heeg, M. E. Hawley, W. Zander, J. Schubert, P. Schiffer, D. A. Muller, L. Maritato, and D. G. Schlom, Effect of biaxial strain on the electrical and magnetic properties of (001) $\mathrm{La}_{0.7} \mathrm{Sr}_{0.3} \mathrm{MnO}_{3}$ thin films, Appl. Phys. Lett. 95, 112504 (2009).

[50] M. Medarde, M. T. Fernández-Díaz, and P. Lacorre, Longrange charge order in the low-temperature insulating phase of $\mathrm{PrNiO}_{3}$, Phys. Rev. B 78, 212101 (2008).

[51] X. Granados, J. Fontcuberta, X. Obradors, L. Mañosa, and J. Torrance, Metallic state and the metal-to-insulator transition of $\mathrm{NdNiO}_{3}$, Phys. Rev. B 48, 11666 (1993).

[52] U. Staub, G. I. Meijer, F. Fauth, R. Allenspach, J. G. Bednorz, J. Karpinski, S. M. Kazakov, L. Paolasini, and F. d'Acapito, Direct Observation of Charge Order in an Epitaxial $\mathrm{NdNiO}_{3}$ Film, Phys. Rev. Lett. 88, 126402 (2002).

[53] R. K. Zheng, H.-U. Habermeier, H. L. W. Chan, C. L. Choy, and H. S. Luo, Effects of substrate-induced strain on transport properties of $\mathrm{LaMnO}_{3+\delta}$ and $\mathrm{CaMnO}_{3}$ thin films using ferroelectric poling and converse piezoelectric effect, Phys. Rev. B 81, 104427 (2010).

[54] T. Wu, P. Zhao, M. Bao, A. Bur, J. L. Hockel, K. Wong, K. P. Mohanchandra, C. S. Lynch, and G. P. Carman, Domain engineered switchable strain states in ferroelectric (011) $\left[\mathrm{Pb}\left(\mathrm{Mg}_{1 / 3} \mathrm{Nb}_{2 / 3}\right) \mathrm{O}_{3}\right]_{(1-\mathrm{x})}-\left[\mathrm{PbTiO}_{3}\right]_{\mathrm{x}}(\mathrm{PMN}-\mathrm{PT}$, $x \approx 0.32$ ) single crystals, J. Appl. Phys. 109, 124101 (2011).

[55] T. X. Nan, M. Liu, W. Ren, Z.-G. Ye, and N. X. Sun, Voltage control of metal-to-insulator transition and non-volatile ferroelastic switching of resistance in $\mathrm{VO}_{\mathrm{x}} / \mathrm{PMN}-\mathrm{PT}$ heterostructures, Sci. Rep. 4, 5931 (2014).

[56] B. Zhi, G. Gao, H. Xu, F. Chen, X. Tan, P. Chen, L. Wang, and $\mathrm{W}$. $\mathrm{Wu}$, Electric-field-modulated nonvolatile resistance switching in $\mathrm{VO}_{2} / \mathrm{PMN}-\mathrm{PT}(111)$ heterostructures, ACS Appl. Mater. Interfaces 6, 4603 (2014).

[57] M. Liu, J. Hoffman, J. Wang, J. Zhang, B. N. Cheeseman, and A. Bhattacharya, Non-Volatile ferroelastic switching of the Verwey transition and resistivity of epitaxial $\mathrm{Fe}_{3} \mathrm{O}_{4} / \mathrm{PMN}-\mathrm{PT}$ (011), Sci. Rep. 3, 1876 (2013).

[58] Y. Yang, Z. L. Luo, M. M. Yang, H. Huang, H. Wang, J. Bao, G. Pan, Q. Hao, S. Wang, M. Jokubaitis, W. Zhang, G. Xiao, Y. Yao, Y. K. Liu, and X. G. $\mathrm{Li}$, Piezo-strain induced non-volatile resistance states in (011) $-\mathrm{La}_{2 / 3} \mathrm{Sr}_{1 / 3} \mathrm{MnO}_{3} / 0.7 \mathrm{~Pb}\left(\mathrm{Mg}_{2 / 3} \mathrm{Nb}_{1 / 3}\right) \mathrm{O}_{3}-0.3 \mathrm{PbTiO}_{3}$ epitaxial heterostructures, Appl. Phys. Lett. 102, 033501 (2013).

[59] Y. Chen, K. H. Lam, D. Zhou, Q. W. Yue, Y. X. Yu, J. C. Wu, W. B. Qiu, L. Sun, C. Zhang, H. S. Luo, H. L. W. Chan, and J. Y. Dai, High performance relaxor-based ferroelectric single crystals for ultrasonic transducer applications, Sensors 14, 13730 (2014). 